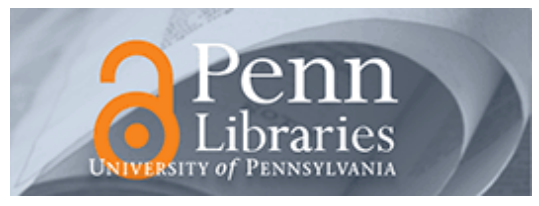

University of Pennsylvania

ScholarlyCommons

Finance Papers

Wharton Faculty Research

2008

\title{
Optimal Decentralized Investment Management
}

Jules van Binsbergen

University of Pennsylvania

Michael W. Brandt

Ralph Koijen

Follow this and additional works at: https://repository.upenn.edu/fnce_papers

Part of the Finance Commons, and the Finance and Financial Management Commons

\section{Recommended Citation}

van Binsbergen, J., Brandt, M. W., \& Koijen, R. (2008). Optimal Decentralized Investment Management. The Journal of Finance, 63 (4), 1849-1895. http://dx.doi.org/10.1111/j.1540-6261.2008.01376.x

At the time of publication, author Jules van Binsbergen was affiliated with Stanford University. Currently, he is a faculty member at the Wharton School at the University of Pennsylvania.

This paper is posted at ScholarlyCommons. https://repository.upenn.edu/fnce_papers/294

For more information, please contact repository@pobox.upenn.edu. 


\title{
Optimal Decentralized Investment Management
}

\author{
Abstract \\ We study an institutional investment problem in which a centralized decision maker, the Chief Investment \\ Officer (CIO), for example, employs multiple asset managers to implement investment strategies in \\ separate asset classes. The $\mathrm{ClO}$ allocates capital to the managers who, in turn, allocate these funds to \\ the assets in their asset class. This two-step investment process causes several misalignments of \\ objectives between the $\mathrm{CIO}$ and his managers and can lead to large utility costs for the $\mathrm{CIO}$. We focus on \\ (1) loss of diversification, (2) unobservable managerial appetite for risk, and (3) different investment \\ horizons. We derive an optimal unconditional linear performance benchmark and show that this \\ benchmark can be used to better align incentives within the firm. We find that the CIO's uncertainty about \\ the managers' risk appetites increases both the costs of decentralized investment management and the \\ value of an optimally designed benchmark. \\ Disciplines \\ Finance | Finance and Financial Management

\section{Comments} \\ At the time of publication, author Jules van Binsbergen was affiliated with Stanford University. Currently, \\ he is a faculty member at the Wharton School at the University of Pennsylvania.
}


NBER WORKING PAPER SERIES

OPTIMAL DECENTRALIZED INVESTMENT MANAGEMENT

Jules H. van Binsbergen

Michael W. Brandt

Ralph S.J. Koijen

Working Paper 12144

http://www.nber.org/papers/w12144

\author{
NATIONAL BUREAU OF ECONOMIC RESEARCH \\ 1050 Massachusetts Avenue \\ Cambridge, MA 02138 \\ March 2006
}

We thank Frank de Jong, Theo Nijman, Anna Pavlova, Bas Werker, and seminar participants at Duke University and Tilburg University for helpful comments. Jules van Binsbergen thanks the Prins Bernhard Cultuurfonds for generous financial support. Binsbergen: Durham, NC 27708. Phone: (919) 660-7636. Email: jules.vanbinsbergen@duke.edu. Brandt: Durham, NC 27708. Phone: (919) 660-1948. Email: mbrandt@duke.edu. Koijen: Tilburg, the Netherlands, 5000 LE. Phone: 31-13-4663238. Email: r.s.j.koijen@tilburguniversity.nl. The views expressed herein are those of the author(s) and do not necessarily reflect the views of the National Bureau of Economic Research.

C2006 by Jules H. van Binsbergen, Michael W. Brandt and Ralph S.J. Koijen. All rights reserved. Short sections of text, not to exceed two paragraphs, may be quoted without explicit permission provided that full credit, including (C) notice, is given to the source. 
Optimal Decentralized Investment Management

Jules H. van Binsbergen, Michael W. Brandt, Ralph S.J. Koijen

NBER Working Paper No. 12144

March 2006

JEL No. G10, G11

\begin{abstract}
$\underline{\text { ABSTRACT }}$
We study a decentralized investment problem in which a CIO employs multiple asset managers to implement and execute investment strategies in separate asset classes. The CIO allocates capital to the managers who, in turn, allocate these funds to the assets in their asset class. This two-step investment process causes several misalignments of objectives between the $\mathrm{CIO}$ and his managers and can lead to large utility costs on the part of the CIO. We focus on i) loss of diversification ii) different appetites for risk, iii) different investment horizons, and iv) the presence of liabilities. We derive an optimal unconditional linear performance benchmark and show that this benchmark can be used to better align incentives within the firm. The optimal benchmark substantially mitigates the utility costs of decentralized investment management. These costs can be further reduced when the $\mathrm{CIO}$ can screen asset managers on the basis of their risk appetites. Each manager's optimal level of risk aversion depends on the asset class he manages and can differ substantially from the CIO's level of risk aversion.
\end{abstract}

Jules H. van Binsbergen

The Fuqua School of Business

Duke University

Box 90120

Durham, NC 27708-0120

jules.vanbinsbergen@duke.edu

Michael W. Brandt

The Fuqua School of Business

Duke University

Box 90120

Durham, NC 27708-0120

and NBER

mbrandt@duke.edu

Ralph S.J. Koijen

Finance Department

Tilburg University

Room B 914

P.O. Box 90153

5000 LE, Tilburg

THE NETHERLANDS

r.s.j.koijen@tilburguniversity.nl 


\section{Introduction}

The investment management divisions of banks, mutual funds, and pension funds are predominantly structured around asset classes such as equities, fixed income, and alternative investments. To achieve superior returns, either through asset selection or market timing, gathering information about specific assets and capitalizing on the acquired informational advantage requires a high level of specialization. This induces the Chief Investment Officer (CIO) of the firm, for example, to pick asset managers who are specialized in one and only one asset class and to delegate portfolio decisions to these specialists. The consequence of this delegation is that asset allocation decisions are made in at least two stages. In the first stage the $\mathrm{CIO}$ allocates capital to the different asset classes, each managed by a different asset manager. In the second stage each manager decides how to allocate the funds made available to him to the assets within his class. This two-stage process can induce several misalignments of incentives that may lead to large utility costs on the part of the CIO. In this paper we show that designing appropriate return benchmarks and/or optimally selecting the risk aversion levels of the managers can substantially reduce these costs.

We focus on the following important, yet not exhaustive, list of misalignments of incentives. First, the two-stage process can lead to severe diversification losses. The unconstrained (single-step) solution to the mean-variance optimization problem is likely different from the optimal linear combination of mean-variance efficient portfolios in each asset class, as pointed out by Sharpe (1981) and Elton and Gruber (2004). Second, there may be considerate differences in appetites for risk between the CIO and each of the asset managers. Third, the investment horizons of the asset managers and of the CIO may be different. Since the managers are usually compensated on an annual basis, their investment horizon is generally relatively short. The CIO, in contrast, may have a much longer investment horizon. Finally, when the investment management firm has to meet certain liabilities (for example pensions or insurance claims) this affects the optimal portfolio choice of the CIO, but not those of unconstrained asset managers.

In practice, the performance of each asset manager is measured against a benchmark comprised of a large number of assets within his class. So far, in the literature, the main purpose of these benchmarks has been to disentangle the effort and achievements of the asset manager from the investment opportunity set available to him. However, in this paper we show that an optimally designed unconditional benchmark can also serve to improve the alignment of incentives within the firm and to substantially mitigate the utility costs of decentralized investment management.

Our results provide a different perspective on the use of performance benchmarks. Admati 
and Pfleiderer (1997) take a realistic benchmark as given and show that when an investment manager uses the conditional return distribution in his investment decisions, restricting him by an unconditional benchmark distorts incentives. ${ }^{1}$ In their framework, this distortion can only be prevented by setting the benchmark equal to the minimum variance portfolio. We show that the negative aspect of unconditional benchmarks can (at least partially) be offset by the role of unconditional benchmarks in aligning other incentives, such as diversification, risk preferences, investment horizons, and liabilities.

We use a stylized representation of an investment management firm to quantify the costs of the misalignments for both constant and time-varying investment opportunities. We assume that the $\mathrm{CIO}$ acts in the best interest of a large group of beneficiaries of the assets under management whereas the investment managers only wish to maximize their personal compensation. Using only two asset classes (bonds and stocks) and three assets per class (government bonds, Baa corporate bonds, and Aaa corporate bonds in the fixed income class and growth stocks, intermediate, and value stocks in the equities class) the utility costs can range from 50 to 300 basis points per year. Therefore, we argue that decentralization has a first order effect on the performance of investment management firms.

We demonstrate that when the investment opportunity set is constant, the CIO can fully align incentives through am unconditional benchmark consisting only of assets in each manager's own asset class. In other words, cross-benchmarking is not required. Optimally selecting the risk aversion of the asset managers can also mitigate the costs of decentralized asset management, but can not fully eliminate them when asset classes are correlated. Furthermore, we derive the perhaps counter-intuitive result that the optimal level of risk aversion of the asset managers, from the perspective of the CIO, can substantially differ from the risk aversion of the CIO.

When investment opportunities are time-varying, an unconditional (passive) benchmark can still substantially, yet not fully, mitigate the utility costs of decentralized investment management. Optimally selecting the risk aversion of the managers can partially achieve the same goal. Finally, we show that even when the CIO optimally selects the risk attitudes of the investment managers, an optimally designed performance benchmark reduces further the costs of decentralized investment management by 10 to 30 percent, depending on the CIO's investment horizon.

The negative impact of decentralized investment management on diversification was first noted by Sharpe (1981), who shows that if the CIO has rational expectations about the portfolio choices of the investment managers, he can choose his investment weights such

\footnotetext{
${ }^{1}$ See also Basak, Shapiro, and Teplá (2005).
} 
that diversification is at least partially restored. However, this optimal linear combination of mean-variance efficient portfolios within each asset class usually still differs from the optimally diversified portfolio over all assets. To restore diversification further, Sharpe (1981) suggests that the CIO imposes investment rules on one or both of the investment managers to solve an optimization problem that includes the covariances between assets in different asset classes. Elton and Gruber (2004) show that it is possible to overcome the loss of diversification by providing the asset managers with investment rules that they are required to implement. The asset managers can then implement the CIO's optimal strategy without giving up their private information.

Both investment rules described above interfere with the asset manager's desire to maximize his individual performance on which his compensation depends. Furthermore, when the investment choices of the managers are not always fully observable, these ad hoc rules are not enforceable. Instead, we propose to change the incentives of the managers by introducing a return benchmark against which they are evaluated for the purpose of their compensation. When this benchmark is implemented in the right way, it is in the manager's own interest to follow investment strategies which are (more) in line with the objectives of the CIO. In Section 2, we assume that investment opportunities are constant. This allows us to the focus on loss of diversification and on differences in preferences in a parsimonious framework. We then add market-timing skill and horizon effects in Section 3 and study the role of liabilities in Section 4.

Perhaps one of the most interesting questions is why the CIO should hire multiple asset managers to begin with. Sharpe (1981) motivates the decision to employ multiple managers by exploiting their specialization or by diversifying among asset managers. Barry and Starks (1984) argue that risk sharing considerations may also imply that it is optimal to employ more than one manager. In Section 3, investment opportunities are time-varying which is motivated by the increasing empirical evidence that equity and in particular bond returns are to some extent predictable. ${ }^{2}$ This allows skilled managers to implement active strategies which generate, when compared to unconditional (passive) return benchmarks, alphas. This specific interpretation of alpha may seem unconventional, but it avoids the question of whether asset managers do or do not have private information. Treynor and Black (1973), Admati and Pfleiderer (1997), and Elton and Gruber (2004) assume that managers can generate alpha, but do not explicitly model how managers do so. Cvitanić, Lazrak, Martellini, and Zapatero (2005) assume that the investor is uncertain about the

\footnotetext{
${ }^{2}$ See, for example, Ang and Bekaert (2005), Lewellen (2004), Campbell and Yogo (2005), and Torous, Valkanov, and Yan (2005) for stock return predictability, and Dai and Singleton (2002) and Cochrane and Piazzesi (2005) for bond return predictability.
} 
alpha of the manager and derive the optimal policy in that case. We explicitly model the time-variation in investment opportunities and assume that the resulting predictability can be exploited by skilled managers to generate value.

Apart from the tactical aspect of return predictability, time-variation in risk premia can also have serious strategic consequences. After all, when asset returns are predictable, the optimal portfolio choice of the CIO depends on his investment horizon. ${ }^{3}$ It then requires dynamic optimization to find the optimal composition of the CIO's portfolio. The resulting portfolio choice is referred to as strategic as opposed to myopic (or tactical). The differences between the strategic and myopic portfolio weights are called hedging demands as they hedge against future changes in the investment opportunity set. These hedging demands are usually more pronounced for longer investment horizons of the CIO. As the remuneration schemes of investment managers are generally based on a relatively short period, their portfolio weights will be virtually myopic. The CIO, in contrast, usually has a long-term investment horizon. This leads to a third misalignment of incentives.

When unconditional benchmarks are used to overcome costs induced by differences in investment horizons, a key question is whether (i) the benchmark and/or (ii) the strategic allocation to the different asset classes exhibit horizon effects. Most strategic asset allocation papers take a centralized perspective as a starting point in which the tactical and strategic aspects are in perfect harmony. Once investment management is decentralized, tactical and strategic motives are separated. We show that both the strategic allocation, i.e., the allocation to the various asset classes, and the optimal benchmarks exhibit strong horizon effects. In fact, once investment managers are not constrained by a benchmark, the horizon effects in the strategic allocation are less pronounced, implying that the strategic allocation and optimal benchmarks should be designed jointly.

Finally, our paper also relates to the standard principal-agent literature in which the agent's effort is unobservable. In the delegated portfolio management context, the agent should exert effort to gather the information needed to make the right portfolio decisions, as explored by Ou-Yang (2003). ${ }^{4}$ we abstract from explicitly modeling the effort choices of the asset managers. Instead, the managers add value by timing the market, which we assume the CIO cannot do. The agency problem arises because the investment managers, whose actions are not always fully observable, wish to maximize their annual compensation, whereas the CIO acts in the best interest of the beneficiaries of the firm. When designing

\footnotetext{
${ }^{3}$ See, for instance, Kim and Omberg (1996), Brennan, Schwartz, Lagnado (1997), Campbell and Viceira (1999), Brandt (1999,2005), Aït-Sahalia and Brandt (2001), Campbell, Chan, and Viceira (2003), and Jurek and Viceira (2005), and Sangvinatsos and Wachter (2005).

${ }^{4}$ Stracca (2005) provides a recent survey of the theoretical literature on delegated portfolio management.
} 
the benchmark, the CIO faces a tradeoff between (i) letting the investment managers realize the gains from market timing and (ii) correcting the misalignments of incentives described above. As a result, the investment problem we solve is non-trivially harder than the problem with a CIO and a single investment manager. After all, the strategic allocation of the CIO now results from a joint optimization over the benchmark and the strategic allocation

to the asset managers. For ease of exposition, we confine attention to a tractable CRRA preference structure and a realistic linear class of performance benchmarks which are assumed to satisfy the participation constraint of the asset managers. Our work also relates to the organizational literature of Dessein, Garicano, and Gertner (2005), who investigate a general manager (in our case the CIO) who attempts to achieve a common goal, while providing strong performance-linked compensation schemes to specialists (in our case the investment managers) to overcome the moral hazard problem. They show that to achieve the common goal, individual incentives may have to be weakened. A common way to align incentives is to give the managers a share in each other's output. Our results indicate that in the portfolio management setting, cross-benchmarking, where the benchmarks of the different asset managers include assets from other classes, is not required.

The paper proceeds as follows. In Section 2 the model is presented in a financial market with constant investment opportunities. Section 3 extends the financial market by allowing for time-variation in expected returns. Section 4 treats several extensions of the basic model including short sales constraints, risk constraints, and liabilities. Section 5 concludes.

\section{Constant investment opportunities}

\subsection{Financial market and preferences}

We assume that the financial market contains $2 k+1$ assets with prices denoted by $S_{i}$, $i=0, \ldots, 2 k$. The first asset, $S_{0}$, is a riskless cash account, which evolves as:

$$
\frac{\mathrm{d} S_{0 t}}{S_{0 t}}=r \mathrm{~d} t
$$

where $r$ denotes the (constant) instantaneous short rate. The remaining $2 k$ assets are risky. We assume that the dynamics of the risky assets are given by geometric Brownian motions. For $i=1, \ldots, 2 k$, we have:

$$
\frac{\mathrm{d} S_{i t}}{S_{i t}}=\left(r+\sigma_{i}^{\prime} \Lambda\right) \mathrm{d} t+\sigma_{i}^{\prime} \mathrm{d} Z_{t}
$$


where $\Lambda$ denotes a $2 k$-dimensional vector of, for now, constant prices of risk and $Z$ is a $2 k$-dimensional vector of independent standard Brownian shocks. All correlations between the asset returns are captured by the volatility vectors $\sigma_{i}$. The volatility matrix of the first $k$ assets is given by $\Sigma_{1}=\left(\sigma_{1}, \ldots, \sigma_{k}\right)^{\prime}$ and for the second $k$ assets by $\Sigma_{2}=\left(\sigma_{k+1}, \ldots, \sigma_{2 k}\right)^{\prime}$.

We consider a parsimonious representation of an investment management firm in which a CIO acts in the best interest of the beneficiaries of the firm. The CIO employs two asset managers who, independently of each other, decide on the optimal composition of their portfolios using a subset of the assets available. The first asset manager has the mandate to manage the first $k$ assets and the second manager has the mandate to invest in the remaining $k$ assets. We explicitly model the preferences of the $\mathrm{CIO}$ and of the investment managers. Initially, the preference structures are assumed to be common knowledge. We postulate that the preferences of the CIO and of the two asset managers can be represented by a CRRA utility function, so that each solves the problem:

$$
\max _{\left(x_{i s}\right)_{s \in\left[t, T_{i}\right]}} \mathbb{E}_{t}\left(\frac{1}{1-\gamma_{i}} W_{T_{i}}^{1-\gamma_{i}}\right),
$$

where $\gamma_{i}$ denotes the coefficient of relative risk aversion, $T_{i}$ the investment horizon, and $i=1,2, C$ refers to the two asset managers and the CIO, respectively. The vector $x_{i}$ denotes the optimal portfolio weights in the different assets available to agent $i$. According to equation (3), the preferences of the $\mathrm{CIO}$ and the investment managers may be conflicting along two dimensions. First, the risk attitudes are likely to be mismatched. Second, the investment horizon used in determining the optimal portfolio choices are potentially different. The remuneration schemes of asset managers usually induce short, say annual, investment horizons. This form of managerial myopia tends to be at odds with the, generally, more long-term perspective of the CIO. The difference in horizons is particularly important for CIOs with long-term mandates from pension funds and life insurers.

In this section, we assume that investment opportunities are constant. Section 2.2 solves for the optimal portfolio choice when investment management is centralized, implying that the CIO optimizes himself over the complete asset menu. In this case, all beforementioned misalignments of incentives are naturally absent. However, when the investment management firm has a rich investment opportunity set and a substantial amount of funds under management, centralized investment management becomes infeasible. In Section 2.3, we therefore introduce asset managers for each asset class assuming that the asset managers operate unconstrained by a benchmark. In Section 2.4, the asset managers are then evaluated relative to a performance benchmark, and we show how to design this benchmark optimally. 
Finally, in Section 2.5 we show how to optimally select risk attitudes of the investment managers in absence of a benchmark. The CIO can pick investment managers from a continuum of investment managers characterized by different risk attitudes. The derivation of the main results is provided in Appendix A.

\subsection{Centralized problem}

As a point of reference, we consider first the centralized problem in which the CIO decides upon the optimal weights in all $2 k+1$ assets. The instantaneous volatility matrix of the risky assets is given by:

$$
\Sigma=\left[\begin{array}{c}
\Sigma_{1} \\
\Sigma_{2}
\end{array}\right] .
$$

If the CIO makes allocation decisions at the asset level and only delegates his decision to the managers for execution, the optimal portfolio is given by:

$$
x_{C}=\frac{1}{\gamma_{C}}\left(\Sigma \Sigma^{\prime}\right)^{-1} \Sigma \Lambda
$$

with the remainder, $1-x_{C}^{\prime} \iota$, invested in the cash account. The utility derived by the CIO from implementing this optimal allocation is:

$$
J_{1}\left(W, \tau_{C}\right)=\frac{1}{1-\gamma_{C}} W^{1-\gamma_{C}} \exp \left(a_{1} \tau_{C}\right),
$$

with $\tau_{C}=T_{C}-t$ and

$$
a_{1}=\left(1-\gamma_{C}\right) r+\frac{1}{2} \frac{1-\gamma_{C}}{\gamma_{C}} \Lambda^{\prime} \Sigma^{\prime}\left(\Sigma \Sigma^{\prime}\right)^{-1} \Sigma \Lambda .
$$

When investment opportunities are constant, the CIO's optimal allocation is independent of the investment horizon, as shown by Merton $(1969,1971)$.

The asset set contains six risky assets. We assume that only the CIO has access to a cash account and that, in line with Brennan (1993) and Gómez and Zapatero (2003), the asset managers cannot borrow. The first three assets are fixed income portfolios, namely a government bond index and two Lehman corporate bond indices with Aaa and Baa ratings. The remaining three assets are equity portfolios made up of firms sorted into value, intermediate, and growth categories based to their book-to-market ratio. The model is estimated by maximum likelihood using data from December 1973 through November 
2004. The nominal short rate is set to five percent per annum. Finally, to ensure statistical identification of the elements of the volatility matrix, we assume that $\Sigma$ is lower triangular.

The estimation results are provided in Table 1. Panel A shows the estimates of the parameters $\Lambda$ and $\Sigma$. Panel $B$ shows the implied instantaneous expected return and correlations between the assets. In the fixed income asset class, we find an expected return spread of one percent between corporate bonds with Baa versus Aaa rating. In the equities asset class, we estimate a high value premium of 4.8 percent. The correlations within asset classes are high, between 80 and 90 percent. Furthermore, there is clear dependence between asset classes, which, as we show more formally later, implies that the two-stage investment process leads to inefficiencies.

Figure 1 portrays the optimal centralized asset allocation of the CIO for a constant investment opportunity set. The figure shows the CIO's mean-variance (MV) frontier, the tangency portfolio, and the optimal portfolios for risk aversions of two, five, and 10. The tangency portfolio has the following portfolio weights: 10 percent in government bonds, 52 percent in corporate Baa bonds, -18 percent in corporate Aaa bonds, -66 percent in growth stocks, 30 percent in intermediate, and 93 percent in value stocks. It has an expected return of 16 percent with a standard deviation of 14 percent per year.

\subsection{Decentralized problem without a benchmark}

We now solve the decentralized problem in which the first asset manager has the mandate to decide on the first $k$ assets and the second asset manager manages the remaining $k$ assets. Neither of the asset managers has access to a cash account. If they did, they could hold highly leveraged positions or large cash balances, which is undesirable from the CIO's perspective. ${ }^{5}$ The CIO allocates capital to the two asset managers and invests the remainder, if any, in the cash account.

The optimal portfolio of asset manager $i$ when he is not constrained by a benchmark is given by:

$$
x_{i}^{N B}=\frac{1}{\gamma_{i}} x_{i}+\left(1-\frac{x_{i}^{\prime} \iota}{\gamma_{i}}\right) x_{i}^{M V},
$$

with

$$
x_{i}=\left(\Sigma_{i} \Sigma_{i}^{\prime}\right)^{-1} \Sigma_{i} \Lambda \text { and } x_{i}^{M V}=\frac{\left(\Sigma_{i} \Sigma_{i}^{\prime}\right)^{-1} \iota}{\iota^{\prime}\left(\Sigma_{i} \Sigma_{i}^{\prime}\right)^{-1} \iota} .
$$

\footnotetext{
${ }^{5}$ A similar cash constraint has been imposed in investment problems with a CIO and a single investment manager (e.g. Brennan (1993) and Gómez and Zapatero (2003)).
} 
The optimal portfolio of the asset managers can be decomposed into two components. The first component, $x_{i}$, is the standard myopic demand which optimally exploits the riskreturn trade-off. The second component, $x_{i}^{M V}$, minimizes the instantaneous return variance, and is therefore labeled the minimum variance portfolio. The minimum variance portfolio substitutes for the riskless asset in the optimal portfolio of the asset manager. These two portfolios are then balanced by the risk attitude of the asset manager.

The CIO has to decide how to allocate capital to the two asset managers as well as to the cash account. We call this decision the strategic asset allocation. The investment problem of the CIO is of the same form as in the centralized problem, but with a reduced asset set. In the centralized setting the CIO has access to $2 k+1$ assets. In the decentralized case, each asset manager combines the $k$ assets in his class to form his preferred portfolio. The CIO can then only choose between these two portfolios and the cash account. The instantaneous volatility matrix of the two risky portfolios available to the CIO is given by:

$$
\bar{\Sigma}=\left[\begin{array}{l}
x_{1}^{N B^{\prime}} \Sigma_{1} \\
x_{2}^{N B^{\prime}} \Sigma_{2}
\end{array}\right] .
$$

Thus, the optimal strategic allocation of the CIO to the two asset managers is given by:

$$
x_{C}=\frac{1}{\gamma_{C}}\left(\bar{\Sigma} \bar{\Sigma}^{\prime}\right)^{-1} \bar{\Sigma} \Lambda,
$$

with the remainder $1-x_{C}^{\prime} \iota$ invested in the cash account.

Throughout the paper, utility costs of decentralized investment management are calculated at the centralized level. In other words, we use the value function of the CIO (the principal) to measure the welfare losses.

The value function of the $\mathrm{CIO}$ with decentralization is given by:

$$
J_{2}\left(W, \tau_{C}\right)=\frac{1}{1-\gamma_{C}} W^{1-\gamma_{C}} \exp \left(a_{2} \tau_{C}\right),
$$

with $\tau_{C}=T_{C}-t$ and

$$
a_{2}=\left(1-\gamma_{C}\right) r+\frac{1}{2} \frac{1-\gamma_{C}}{\gamma_{C}} \Lambda^{\prime} \bar{\Sigma}^{\prime}\left(\bar{\Sigma} \bar{\Sigma}^{\prime}\right)^{-1} \bar{\Sigma} \Lambda .
$$

It is straightforward to show that the value function in equation (6) (the centralized problem) is larger than or equal to the value function in equation (12) (the decentralized problem). This follows from the fact that the two-stage asset allocation procedure reduces the asset set 
of the CIO as explained above. The CIO can only allocate funds between the two managers which does not provide sufficient flexibility to achieve the first-best solution.

The two-stage asset allocation results in the first-best outcome only when the asset managers already happen to implement the proper relative weights within their asset classes. In this case, the CIO can use the strategic allocation to scale up the asset manager's weights to the optimal firm-level allocation. A set of sufficient conditions for this to hold is given by:

$$
\begin{aligned}
\Sigma_{1} \Sigma_{2}^{\prime} & =0_{k \times k} \\
x_{i}^{\prime} \iota & =\gamma_{i}
\end{aligned}
$$

with $i=1,2$. Note that even when asset classes are independent, i.e., condition (14) holds, the first best solution is generally not attainable. This is because of the absence of a cash account, which implies that the managers allocate their funds to the (efficient) tangency portfolio and the (inefficient) minimum variance portfolio. Condition (15) ensures that the investment in the minimum variance portfolio equals zero. If both conditions are satisfied, the CIO's optimal strategic allocation to the managers is given by $\gamma_{i} / \gamma_{C}, i=1,2$.

Figure 2 illustrates the solution of the decentralized portfolio problem for a CIO who hires two investment managers with equal risk aversion of two in the top graph, five in the middle graph, and 10 in the bottom graph. Each plot shows the MV frontier of the bond manager, the MV frontier of the stock manager, and the CIO's optimal linear combination of these two frontiers. As we argued above, the decentralized MV frontier lies within the centralized MV frontier. Furthermore, the decentralized MV frontier crosses the MV frontier for stocks at the preferred portfolio of the stock manager and it crosses the MV for bonds at the portfolio chosen by the bond manager.

The figure also shows the portfolio choices of the CIO for both the centralized and decentralized scenario for risk aversion of two in the top graph, five in the middle graph, and finally 10 in the bottom graph. The results clearly shows that the CIO invests more conservatively in the decentralized case. In fact, it can be shown in general that the optimal decentralized portfolio is always more conservative than the optimal centralized portfolio.

In Figure 3, we show the welfare losses caused by decentralized investment management for various combinations of risk attitudes of the asset managers. The coefficient of relative risk aversion of the CIO equals $\gamma_{C}=5$ in Panel A and $\gamma_{C}=10$ in Panel B. We define the welfare loss as the decrease in the annualized certainty equivalent return at the firm-level. Interestingly, this loss is not minimized when the risk aversion of the asset managers is equal to that of the CIO. In fact, the cost of decentralized investment management is minimized 
for a risk aversion of 3.3 for the stock manager and 5.7 for the bond manager, regardless of the risk aversion of the CIO. Even though the location of the minimum is not dependent of the risk aversion of the CIO (to be shown formally in Section 2.5), the utility loss incurred obviously is. When the risk aversion of the CIO equals five, the diversification losses are eight basis points per year in terms of certainty equivalents. This number drops to four basis points when the risk aversion of the CIO equals 10 because he moves out of risky assets and into the riskless asset. The welfare loss can increase to 80-100 basis points even in this simple example for different risk attitudes of the investment managers. Finally, note that when the CIO is forced to hire a bond manager who does not have the optimal risk aversion level, this may influence the CIO's preferred choice of stock manager and vice versa.

Figure 4 shows the portfolio compositions of the bond manager in Panel A and of the stock manager in Panel B as functions of their risk aversion. Recall that the managers do not have access to a riskless asset. Figure 5 shows the fraction of total risky assets that is allocated to the stock manager as a function of his (and the bond manager's) risk aversion. The bond manager receives one minus this allocation. The allocation of capital between the riskless and the risky assets depends on the risk aversion of the CIO and is not shown.

\subsection{Decentralized problem with a benchmark}

We now consider the decentralized investment problem in which the CIO designs a performance benchmark for each of the investment managers in an attempt to align incentives. We restrict attention to benchmarks in the form of portfolios which can be replicated by the asset managers. This restriction implies that only the assets of the particular asset class are used and that the benchmark contains no cash position. That

is, there is no possibility and, as we show later, no need for cross-benchmarking. We denote the value of the benchmark of manager $i$ at time $t$ by $B_{i t}$ and the weights in the benchmark portfolio for asset class $i$ by $\beta_{i}$. The evolution of benchmark $i$ is therefore given by:

$$
\frac{\mathrm{d} B_{i t}}{B_{i t}}=\left(r+\beta_{i}^{\prime} \Sigma_{i} \Lambda\right) \mathrm{d} t+\beta_{i}^{\prime} \Sigma_{i} \mathrm{~d} Z_{t}
$$

with $\beta_{i}^{\prime} \iota=1$, for $i=1,2$.

We postulate that the asset managers derive utility from the ratio of assets to the value of the benchmark. They face the problem:

$$
\max _{\left(x_{i s}\right)_{s \in\left[t, T_{i}\right]}} \mathbb{E}_{t}\left(\frac{1}{1-\gamma_{i}}\left(\frac{W_{i T}}{B_{i T}}\right)^{1-\gamma_{i}}\right)
$$


This preference structure can be motivated in several ways. First, the remuneration schemes of asset managers usually contain a component which depends on the performance relative to a benchmark. This is captured in our model by specifying preferences over the ratio of funds under management to the value of the benchmark, in line with Browne $(1999,2000)$. Second, investment managers often operate under risk constraints. An important way to measure risk attributable to manager $i$ is tracking error volatility. The tracking error is usually defined as the return differential of the funds under management and the benchmark. Taking logs of the ratio of wealth to the benchmark provides the tracking error in log returns. Third, for investment management firms that need to account for liabilities, like pension funds and life insurers, supervisory bodies often summarize the financial position by the ratio of assets to liabilities, the so-called funding ratio as further described in van Binsbergen and Brandt (2006). Hence, the ratio of wealth to the benchmark (liabilities) can be interpreted as a reasonable summary statistic of relative performance. ${ }^{6}$

When the performance of asset manager $i$ is measured relative to the benchmark, his optimal portfolio is given by:

$$
x_{i}^{B}=\frac{1}{\gamma_{i}} x_{i}+\left(1-\frac{1}{\gamma_{i}}\right) \beta_{i}+\frac{1}{\gamma_{i}}\left(1-x_{i}^{\prime} \iota\right) x_{i}^{M V},
$$

where $x_{i}$ and $x_{i}^{M V}$ are given in equation (9). This portfolio differs from the optimal portfolio in absence of a benchmark in two important respects. First, the optimal portfolio contains a component which replicates the composition of the benchmark portfolio. It is exactly this response of the investment manager which allows the CIO to optimally design a benchmark to align incentives. Note that the benchmark weights enter the optimal portfolio linearly. Second, when the coefficient of relative risk aversion, $\gamma_{i}$, tends to infinity, the asset manager tracks the benchmark exactly. Hence, the benchmark is considered to be the riskless asset from the perspective of the asset manager.

The CIO has to optimally design the two benchmark portfolios and has to determine the allocation to the two asset managers as well as to the cash account. It is important to notice that $x_{i}^{B}=x_{i}^{N B}$ when $\beta_{i}=x_{i}^{M V}$. That is, the optimal portfolio with and without performance benchmark coincide when the benchmark portfolio equals the minimum

\footnotetext{
${ }^{6}$ In addition, Stutzer (2003a) and Foster and Stutzer (2003) show that when the optimal portfolio is chosen so that the probability of under-performance tends to zero as the investment horizon goes to infinity, the portfolio which maximizes the probability decay rate solves a criterium similar to power utility with two main modifications. First, the investor's preferences involve the ratio of wealth over the benchmark. Second, the investor's coefficient of relative risk aversion depends on the investment opportunity set. This provides an alternative interpretation of preferences over the ratio of wealth to the benchmark as well as different coefficients of relative risk aversion for the various asset classes.
} 
variance portfolio. As such, the benchmark can only reduce the welfare costs of decentralized investment management. More importantly, when investment opportunities are constant, the benchmark can be designed so that all inefficiencies are eliminated. The composition of the optimal benchmark which leads to the optimal allocation of the centralized investment problem is given by:

$$
\beta_{i}=x_{i}^{M V}+\frac{\gamma_{i}}{\gamma_{i}-1}\left(\frac{x_{i}^{C}}{x_{i}^{C \prime} \iota}-x_{i}^{N B}\right),
$$

where $x_{i}^{C}$ are the optimal weights for the assets under management by manager $i$ when the CIO controls all assets as given in (5) and $x_{i}^{N B}$ is given in (8). The benchmark weights sum to one because of the restriction that the benchmark cannot contain a cash position.

The two components of the optimal benchmark portfolio have a natural interpretation. The first component is the minimum variance portfolio. As we pointed out above, once the benchmark portfolio coincides with the minimum variance portfolio, the benchmark does not affect the manager's optimal portfolio. The second component, however, corrects the manager's portfolio choice to align incentives. If the relative weights of the CIO and the portfolio of the manager without a benchmark (i.e., $x_{i}^{N B}$ ) coincide, there is no need to influence the manager's portfolio and the second term is zero. However, when the CIO optimally allocates a larger share of capital to a particular asset in class $i$, the benchmark will contain a positive position in this asset, if $\gamma_{i}>1$. The ratio before the second component accounts for the manager's preferences. If the manager is more aggressive (i.e., $\gamma_{i} \rightarrow 1$ ), the benchmark weights are more extreme as the manager is less sensitive to benchmark deviations. If the investor becomes very conservative (i.e., $\gamma \rightarrow \infty$ ), we get $x_{i}^{N B}=x_{i}^{M V}$ and the benchmark coincides with the relative weights of the CIO. In this way, the benchmark is an excellent instrument to adjust the relative portfolio weights chosen by the asset managers.

Finally, the CIO uses the strategic allocation to the two asset managers to implement the optimal firm-level allocation. The optimal weight given to each manager is given by $x_{i}^{C \prime} \iota$, with $i=1,2$, and the remainder, $1-x_{1}^{C \prime} \iota-x_{2}^{C \prime} \iota$, is invested in the cash account.

Figure 6 shows the composition of the optimal benchmarks for the bond manager in Panel A and for the stock manager in Panel B as functions of their risk aversion. The mechanism through which the benchmark aligns incentives is particularly clear for the fixed income asset class. Without a benchmark, the bond manager invests too aggressively in corporate bonds with Baa rating, whereas the benchmark contains a large short position in the same asset. This reduces the manager's allocation to Baa rated bonds. For Aaa rated bonds, the benchmark provides exactly the opposite incentive. 


\subsection{Optimal selection of investment managers}

The results above show that when investment opportunities are constant, performance benchmarks can be designed such that the decentralized investment problem coincides with the centralized problem and all welfare costs of decentralization are eliminated. As an alternative mechanism to align incentives, the CIO can anticipate the risk appetites of the investment managers when they are hired. To demonstrate this alternative mechanism, we assume in this section that the $\mathrm{CIO}$ can select asset managers from a continuum of managers with different levels of risk aversion. We show that optimally selecting the managers only partially solves the problem, unless the asset classes are uncorrelated.

We solve the decentralized investment management problem in which the CIO not only decides on the strategic allocation to the asset managers, but also on their risk attitudes, $\gamma_{i}$. Equation (8) shows that the optimal portfolio of investment manager $i$ is affine in his risk tolerance $\gamma_{i}^{-1}$. This implies that different risk attitudes induce different weights placed on the tangency portfolio and on the minimum variance portfolio. Consequently, the CIO selecting asset managers on the basis of their risk attitude is equivalent to expanding the asset set from two to four assets. The CIO can in this case manage the tangency portfolios and minimum variance portfolios independently, as well as select the fractions allocated to the different asset classes. Let $\tilde{x}_{C}$ denote the optimal allocation of the CIO when he has four assets at his disposal, namely the two tangency portfolios as well as the two minimum variance portfolios. This optimal (four dimensional) portfolio composition is given by:

$$
\tilde{x}_{C}=\frac{1}{\gamma_{C}}\left(\tilde{\Sigma} \tilde{\Sigma}^{\prime}\right)^{-1} \tilde{\Sigma} \Lambda,
$$

where $\tilde{\Sigma}$ is defined as:

$$
\tilde{\Sigma}=\left[\begin{array}{c}
\left(x_{1}-\left(x_{1}^{\prime} \iota\right) x_{1}^{M V}\right)^{\prime} \Sigma_{1} \\
x_{1}^{M V \prime} \Sigma_{1} \\
\left(x_{2}-\left(x_{2}^{\prime} \iota\right) x_{2}^{M V}\right)^{\prime} \Sigma_{2} \\
x_{2}^{M V \prime} \Sigma_{2}
\end{array}\right] .
$$

In Appendix A we show that this allocation can be implemented by optimally choosing the risk attitudes of the two asset managers and by selecting the strategic allocation of capital to the managers. The optimal coefficients of relative risk aversion are given by:

$$
\gamma_{1}^{*}=\frac{\tilde{x}_{C(2)}}{\tilde{x}_{C(1)}} \quad \text { and } \quad \gamma_{2}^{*}=\frac{\tilde{x}_{C(4)}}{\tilde{x}_{C(3)}} .
$$


The optimal selection of investment managers is independent of the preferences of the CIO. This is because within an asset class only the relative allocations are important since the absolute allocations can be adjusted using the strategic allocation to the asset classes. The optimal strategic allocation $x_{C}$ to the two asset classes is given by:

$$
x_{1 C}=\tilde{x}_{C(2)} \text { and } \quad x_{2 C}=\tilde{x}_{C(4)} .
$$

The corresponding value function of the CIO is:

$$
J_{3}\left(W, \tau_{C}\right)=\frac{1}{1-\gamma_{C}} W^{1-\gamma_{C}} \exp \left(a_{3} \tau_{C}\right)
$$

with $\tau_{C}=T_{C}-t$ and

$$
a_{3}=\left(1-\gamma_{C}\right) r+\frac{1}{2} \frac{1-\gamma_{C}}{\gamma_{C}} \Lambda^{\prime} \tilde{\Sigma}^{\prime}\left(\tilde{\Sigma} \tilde{\Sigma}^{\prime}\right)^{-1} \tilde{\Sigma} \Lambda
$$

Despite the fact that optimally selecting the asset managers according to their risk tolerances mitigates the inefficiencies induced by decentralized asset management, it generally does not lead to the first best outcome, unless the asset classes are uncorrelated (see condition (14)).

In the empirical application we find that the optimal risk aversion for the stock manager is 3.3 while that for the fixed income manager is 5.7. Hence, in this case, the equity class requires a more aggressive investment manager than the fixed income class. The remaining utility cost of decentralized asset management depends on the risk aversion of the CIO and equals eight basis points when $\gamma_{C}=5$ and four basis points when $\gamma_{C}=10$.

\section{Time-varying investment opportunities}

\subsection{Financial market}

In Section 2, investment opportunities are constant through time and there are only two inefficiencies caused by decentralized investment management, namely loss of diversification between asset classes and misalignments in risk attitudes. However, the role of asset managers is rather limited in that they add no value in the form of stock selection or market timing. In this section, we allow investment opportunities, and in particular expected returns, to be time-varying and predicted by a set of common forecasting variables. This setting allows asset managers to implement active strategies which optimally exploit changes in investment opportunities in their respective asset classes. These active strategies can generate alphas when compared to an unconditional (passive) performance benchmark. Thus active asset 
management can be value-enhancing.

This extension of the problem adds several new interesting dimensions to the decentralized investment management problem. First, differences in investment horizons create another misalignment of incentives. CIOs generally act in the long-term interest of the investment management firm, while asset managers tend to be more shortsighted, possibly induced by their remuneration schemes. When the predictor variables are correlated with returns, it is optimal to hedge future time-variation in investment opportunities. ${ }^{7}$ As a consequence, the myopic portfolios held by the asset managers will generally not coincide with the CIO's optimal portfolio which incorporates long-term hedging demands. Second, when a common set of predictor variables affects the investment opportunities in both asset classes, active strategies are potentially correlated. This implies that even if instantaneous returns are uncorrelated, long-term returns can be correlated, which means that the loss of diversification is aggravated. Third, the role of benchmarks is markedly different compared to the case of constant investment opportunities. For the sake of realism, we restrict attention to passive (unconditional) strategies as return benchmarks. As we discussed earlier, Admati and Pfleiderer (1997) show that when the asset manager has private information, an unconditional benchmark can be very costly. After all, the asset managers base their decision on the conditional return distribution, whereas the CIO designs the benchmark using the unconditional return distribution. ${ }^{8}$ In their framework it follows therefore that unless the benchmark is set equal to the minimum variance portfolio, it induces a potentially large efficiency loss. In our model, on the contrary, the benchmark is used to align incentives. We therefore further explore the role of unconditional return benchmarks and their interplay with differences in investment horizons.

We now consider a more general financial market in which the prices of risk, $\Lambda$, can vary over time. More explicitly, we model:

$$
\Lambda(X)=\Lambda_{0}+\Lambda_{1} X
$$

where $X$ denotes an $m$-dimensional vector of de-meaned state variables that capture timevariation in expected returns. Although the state variables are time-varying, we drop the subscript $t$ for notational convenience. All portfolios in this section are indexed with either the state realization, $X$, or the investment horizon, $\tau$, in order to emphasize the conditioning

\footnotetext{
${ }^{7}$ See for instance Kim and Omberg (1996), Campbell and Viceira (1999), Brandt (1999), and Liu (2006).

${ }^{8}$ Although the predictors are publicly observed, we assume that the CIO is time-constrained or not sufficiently specialized to exploit this information. As such, the conditional return distribution remains unknown for the CIO and the conditioning information exploited by the asset managers is equivalent to private information.
} 
information used to construct the portfolio policies.

Most predictor variables used in the literature, such as term structure variables and financial ratios, are highly persistent. In order to accommodate first-order autocorrelation in predictors, we model their dynamics as Ornstein-Uhlenbeck processes:

$$
\mathrm{d} X_{i t}=-\kappa_{i} X_{i t} \mathrm{~d} t+\sigma_{X i}^{\prime} \mathrm{d} Z_{t}
$$

where $Z$ now denotes a $(2 k+m)$-dimensional Brownian motion. The volatility matrix of the $m$ predictors is given by $\Sigma_{X}=\left(\sigma_{X 1}, \ldots, \sigma_{X m}\right)^{\prime}$. Furthermore, we assume again that only the CIO has access to a cash account. Finally, we postulate the same preference structures for the CIO and the asset managers as in Section 2.1.

We estimate the return dynamics using three predictor variables: the short rate, the yield on a 10-year nominal government bond, and the log dividend yield of the equity index. These predictors have been used in strategic asset allocation problems to capture the timevariation in expected returns (see the references in footnote 3 ). The model is estimated by maximum likelihood using data from January 1973 through November 2004. Following Campbell, Chan, and Viceira (2003), we constrain the levels of the predictor variables to match their sample counterparts. The estimation results are presented in Table 2.

The estimates of the unconditional instantaneous expected returns, $\Lambda_{0}$, are similar to the results in Table 1. The second part of the Table 2 describes the responses of the expected returns of the individual assets to changes in the state variables, $\Sigma \Lambda_{1}$. We find that the short rate has a negative impact on the expected returns of all assets except for government bonds. Furthermore, the expected returns of assets in the fixed income class are positively related to the long-term yield, while the expected returns of assets in the equity class are negatively

related to this predictor. The dividend yield is positively related to the expected returns of all assets. The estimates of the autoregressive parameters, $\kappa_{i}$, reflect the high persistence of the predictor variables. Finally, the last part of Table 2 provides the joint volatility matrix of the assets and the predictor variables.

\subsection{Centralized problem}

We first solve again the centralized investment problem in which the CIO manages all assets. This solution serves as a benchmark for the case in which investment management is decentralized. The centralized investment problem with affine prices of risk has been solved by, among others, Liu (2006) and Sangvinatsos and Wachter (2005). We denote the CIO's 
investment horizon by $\tau_{C}$. The optimal allocation to the different assets is given by:

$$
\begin{aligned}
x_{C}\left(X, \tau_{C}\right)= & \frac{1}{\gamma_{C}}\left(\Sigma \Sigma^{\prime}\right)^{-1} \Sigma \Lambda(X)+\ldots \\
& \frac{1}{\gamma_{C}}\left(\Sigma \Sigma^{\prime}\right)^{-1} \Sigma \Sigma_{X}^{\prime}\left(B\left(\tau_{C}\right)+\frac{1}{2}\left(C\left(\tau_{C}\right)+C\left(\tau_{C}\right)^{\prime}\right) X\right),
\end{aligned}
$$

where expressions for $B\left(\tau_{C}\right)$ and $C\left(\tau_{C}\right)$, as well as the derivations of the results in this section are provided in Appendix B. The optimal portfolio contains two components. The first component is the conditional myopic demand which optimally exploits the risk-return trade-off provided by the assets. The second component represents the hedging demands that emerge from the CIO's desire to hedge future changes in the investment opportunity set. This second term reflects the long-term perspective of the CIO. The corresponding value function is given by:

$$
J_{1}\left(W, X, \tau_{C}\right)=\frac{1}{1-\gamma_{C}} W^{1-\gamma_{C}} \exp \left\{A\left(\tau_{C}\right)+B\left(\tau_{C}\right)^{\prime} X+\frac{1}{2} X^{\prime} C\left(\tau_{C}\right) X\right\},
$$

with the coefficients $A, B$, and $C$ provided in Appendix B.

In Figure 7 we illustrate the composition of the optimal portfolio for different investment horizons when the coefficient of relative risk aversion of the CIO equals either $\gamma_{C}=5$ in Panel A or $\gamma_{C}=10$ in Panel B. Focusing first on the fixed income asset class, we find substantial horizon effects for corporate bonds. At short horizons, the CIO optimally tilts the portfolio towards Baa rated corporate bonds and shorts Aaa rated corporate bonds to take advantage of the credit spread. At longer horizons, the fraction invested in Baa rated bonds increases even further, while the allocation to Aaa rated corporate bonds decreases. Switching to the results for the equities asset class, we detect a strong value tilt at short horizons due to the high value premium. The optimal portfolio contains a large long position in value stocks and large short position in growth stocks. However, as the investment horizon increases, the value tilt drops, consistent with the results of Jurek and Viceira (2005). ${ }^{9}$

\footnotetext{
${ }^{9}$ This result is also in line with the findings of Campbell and Vuolteenaho (2004) who explain the value premium by decomposing the CAPM beta into a cash flow beta and a discount rate beta. The cash flow component is highly priced but largely unpredictable. The discount rate component demands a lower price of risk but is to some extent predictable. Campbell and Vuolteenaho (2004) show that growth stocks have a large discount rate beta, whereas value stocks have a large cash flow beta. This implies that, from a myopic perspective, value stocks are more attractive than growth stocks. However, the predictability of growth stock returns implies that long-term returns on these assets are less risky, making them relatively more attractive.
} 


\subsection{Decentralized problem without a benchmark}

We now solve the decentralized problem when the CIO cannot use the benchmark to align incentives. In general, the optimal portfolios of the asset managers depend on both the investment horizon and the state of the economy. However, to make the problem more tractable and realistic, we assume that the investment managers are able to time the market and exploit the time-variation in risk premia, but ignore long-term considerations. That is, asset managers implement the conditional myopic strategy:

$$
x_{i}^{N B}(X)=\frac{1}{\gamma_{i}} x_{i}(X)+\left(1-\frac{x_{i}(X)^{\prime} \iota}{\gamma_{i}}\right) x_{i}^{M V}
$$

with

$$
x_{i}(X)=\left(\Sigma_{i} \Sigma_{i}^{\prime}\right)^{-1} \Sigma_{i} \Lambda(X) \text { and } x_{i}^{M V}=\frac{\left(\Sigma_{i} \Sigma_{i}^{\prime}\right)^{-1} \iota}{\iota^{\prime}\left(\Sigma_{i} \Sigma_{i}^{\prime}\right)^{-1} \iota} .
$$

This particular form of myopia can be motivated by the relatively short-sighted compensation schemes of asset managers. Since the average hedging demands for one-year horizons are negligible, we abstract from the hedging motives in this part of the problem.

The CIO does account for the long-term perspective of the firm through the strategic allocation. However, we assume that the CIO implements a strategic allocation that is unconditional, i.e., independent of the current state. At every point in time, the allocation to the different asset classes is reset towards a constant proportions strategic allocation, as opposed to constantly changing the strategic allocation depending on the state. In order to decide on the strategic allocation, the CIO maximizes the unconditional value function:

$$
\max _{x_{C}\left(\tau_{C}\right)} \mathbb{E}\left(J_{2}\left(W, X, \tau_{C}\right) \mid W\right),
$$

in which $J_{2}$ denotes the conditional value function in the decentralized problem above. Obviously, the CIO's horizon, $\tau_{C}$, influences the choice of the strategic allocation.

To review the setup of this decentralized problem, the asset managers implement active strategies in their asset classes using conditioning information but ignore any long-term considerations. The CIO, in contrast, allocates capital unconditionally to the asset classes, but accounts for the firm's long-term perspective.

In order to determine the unconditional value function, we evaluate first the conditional value function of the CIO, $J_{2}$, for any choice of the strategic allocation. In Appendix B we 
show that the conditional value function is exponentially quadratic in the state variables:

$$
J_{2}\left(W, X, \tau_{C}\right)=\frac{W^{1-\gamma_{C}}}{1-\gamma_{C}} \exp \left\{\left(A\left(\tau_{C}, x_{C}\right)+B\left(\tau_{C}, x_{C}\right)^{\prime} X+\frac{1}{2} X^{\prime} C\left(\tau_{C}, x_{C}\right) X\right\} .\right.
$$

One aspect of the CIO's problem is particularly interesting. The active strategy implemented by the asset managers, $x_{i}^{N B}$, is affine in the predictor variables $X$ :

$$
x_{i}^{N B}(X)=\zeta_{0 i}^{N B}+\zeta_{1 i}^{N B} X
$$

As a consequence, the implied wealth dynamics faced by the CIO are given by:

$$
\frac{\mathrm{d} W_{t}}{W_{t}}=\left(r+\sigma_{W}(X)^{\prime} \Lambda(X)\right) \mathrm{d} t+\sigma_{W}(X)^{\prime} \mathrm{d} Z_{t},
$$

with

$$
\sigma_{W}(X)^{\prime}=x_{1 C}\left(\zeta_{01}^{N B}+\zeta_{11}^{N B} X\right)^{\prime} \Sigma_{1}+x_{2 C}\left(\zeta_{02}^{N B}+\zeta_{12}^{N B} X\right)^{\prime} \Sigma_{2}
$$

Since the asset managers condition their portfolios on the state variables, the CIO has to allocate capital to two assets which exhibit a very particular form of heteroskedasticity. Hence, despite the homoskedastic nature of the financial market, the CIO is confronted with heteroskedastic asset returns in the decentralized investment management problem.

We solve for the optimal strategic asset allocation numerically (see Appendix B for further details). In Figure 8, we present the strategic allocation to the fixed income and equities classes for different investment horizons. The preference parameters are set to $\gamma_{C}=10$ and $\gamma_{1}=\gamma_{2}=5$. The strategic allocation to the asset classes exhibits substantial horizon effects and marginally overweighs equities. Recall that the strategic allocation to asset classes is by construction independent of the state variables because it is unconditional.

Figure 9 provides the annualized utility costs from decentralized asset management for different risk attitudes of the investment managers. The investment horizon equals either $T=1$ year in Panel A or $T=10$ years in Panel B. The utility costs are large and increasing in the horizon of the CIO. For relatively short investment horizons, the costs closely resemble the case with constant investment opportunities, with an order of about 40 to 80 basis points per annum. In contrast, for longer investment horizons, the utility costs are substantially higher, around 200 to 300 basis points per annum. Note also that for different investment horizons the costs of decentralized asset management are minimized for different risk attitudes of the investment managers. The optimal selection of investment managers and the interplay with 
the CIO's horizon is the subject of Section 3.5.

\subsection{Decentralized problem with a benchmark}

We show in Section 2.4 that when investment opportunities are constant, a performance benchmark can be designed to eliminate all inefficiencies induced by decentralized asset management. This section re-examines this issue for the case of time-varying investment opportunities. We restrict attention to unconditional benchmarks, meaning the benchmark is not allowed to depend on the state variables. ${ }^{10}$ Unconditional benchmarks have the advantage that they are easy to implement. Moreover, investment managers following an unconditional benchmark do not have to trade excessively, which could be the case with a conditional benchmark. Of course, conditional benchmarks are more flexible and may therefore reduce further or even eliminate the costs of decentralized asset management.

The performance benchmark of asset manager $i$ is given by a $k$-dimensional vector of unconditional portfolio weights, $\beta_{i}$, with $\beta_{i}^{\prime} \iota=1$. Since the benchmark is chosen unconditionally, asset managers can outperform their benchmark (i.e., generate alpha) by properly incorporating the conditioning information. The benchmark dynamics are:

$$
\frac{\mathrm{d} B_{i t}}{B_{i t}}=\left(r+\beta_{i}^{\prime} \Sigma_{i} \Lambda(X)\right) \mathrm{d} t+\beta_{i}^{\prime} \Sigma_{i} \mathrm{~d} Z_{t}
$$

To solve for the optimal benchmark, we first determine the optimal response of the asset managers to their benchmarks (assuming the same preference structure as in Section 2.4). The optimal conditional myopic strategy of the investment managers with a benchmark is:

$$
x_{i}^{B}(X)=\frac{1}{\gamma_{i}} x_{i}(X)+\left(1-\frac{1}{\gamma_{i}}\right) \beta_{i}+\frac{1}{\gamma_{i}}\left(1-x_{i}(X)^{\prime} \iota\right) x_{i}^{M V},
$$

with $x_{i}(X)$ and $x_{i}^{M V}$ given by equation (31). The CIO chooses the (unconditional) benchmarks and determines the (unconditional) strategic allocation to the asset classes by maximizing the unconditional expectation of the conditional value function:

$$
\max _{x_{C}\left(\tau_{C}\right), \beta_{1}\left(\tau_{C}\right), \beta_{2}\left(\tau_{C}\right)} \mathbb{E}\left(J_{3}\left(W, X, \tau_{C}\right) \mid W\right)
$$

The conditional value function, $J_{3}$, is again exponentially quadratic in the state variables and the coefficients are provided in Appendix B. Note that both the strategic allocation and the benchmarks are allowed to depend on the CIO's horizon.

\footnotetext{
${ }^{10}$ See also Cornell and Roll (2005).
} 
We use numerical methods to solve for the optimal benchmarks and allocations to the two asset classes (see Appendix B for further details). Panel A of Figure 10 shows the optimal performance benchmarks for different investment horizons of the CIO. The CIO's risk aversion equals 10 and the managers' risk aversion is set to five. At short horizons or if the CIO behaved myopically, the optimal benchmarks are similar as when investment opportunities are constant. However, the benchmark portfolios exhibit strong horizon effects. For instance, in the equities asset class, the myopic benchmark reinforces the value tilt already present in the equity manager's (myopic) portfolio. The long-run benchmark, in contrast, anticipates the lower risk in growth stocks and provides an incentive to reduce the value tilt. This illustrates how performance benchmarks can be used to incorporate the CIO's long-term perspective in the short-term portfolio choices of asset managers.

Panel B of Figure 10 provides the corresponding strategic allocation to both asset classes for different investment horizons. Recall that, when investment opportunities are constant, the centralized allocation is always more risky than the decentralized allocation without a benchmark. When investment opportunities are time-varying, we find the initial allocation with a benchmark to be similar (and even somewhat more conservative) to the allocation without a benchmark. However, for longer investment horizons of the CIO, the optimal strategic allocation of the CIO is tilted substantially towards equities.

Figure 11 presents the welfare gains generated by an optimally constructed benchmark. The CIO's coefficient of risk aversion equals 10 and the horizon is set to $T=1$ year in Panel A and $T=10$ years in Panel B. For the one-year horizon, the value added by the benchmark is limited to approximately 20 basis points. However, when the investment horizon increases to 10 years, the benefit of an optimally chosen benchmark increases as the asset managers become less conservative. In addition, for the short investment problem, optimal selection of the asset manager is equally important for the two asset classes. When the CIO has a long-term perspective, optimally choosing the equities manager is far more important.

\subsection{Optimal selection of asset managers and benchmarks}

We consider two mechanisms for aligning incentives for decentralized investment management. Above we showed that choosing a benchmark optimally can mitigate the costs substantially. In this section, we infer the role of optimally selecting the asset managers, focusing first on the case when the CIO does not use a performance benchmark.

When the CIO can select the investment managers on the basis of their risk attitudes, he has the ability to independently manage the conditional tangency portfolios and the conditional minimum variance portfolios. In order to optimally select the managers, the 
CIO maximizes the uncondition value function in equation (32) with respect to both $\gamma_{1}$ and $\gamma_{2}$, as well as the strategic allocation $x_{C}$. When the CIO has a coefficient of relative risk aversion of $\gamma_{C}=5$ and the investment horizon equals 10 years, the optimal risk aversion levels of the fixed income and equity asset managers are given by $\gamma_{1}=2.8$ and $\gamma_{2}=7.8$, respectively. The corresponding cost of decentralized asset management equals 340 basis points per year. Similarly, when the CIO's risk attitude is $\gamma_{C}=10$ and the investment horizon equals 10 years, the optimal risk aversion levels are $\gamma_{1}=2.9$ and $\gamma_{2}=9.6$. The cost of decentralized asset management equals 198 basis points in this case.

Recall that when investment opportunities are constant, the optimal risk attitudes of the asset managers are independent of the risk attitude of the CIO. This result does not carry over to the case with time-varying investment opportunities. For a 10-year investment horizon, Panel B of Figure 12 illustrates that as the risk aversion of the CIO increases, it is optimal to select more conservative investment managers. This effect is particularly pronounced for the equities asset class. Moreover, the manager optimally selected for the equities class is far more conservative than the one optimally selected for the fixed income asset class. In order to illustrate the role of the investment horizon, we present in Panel A of Figure 12 the optimal risk attitudes of the managers when the CIO has an investment horizon of one year. We find in this case that the selection of the optimal investment managers is (almost) independent of the risk aversion of the $\mathrm{CIO}$, in line with the case of constant investment opportunities. These results imply that for long-term investment management firms, such as pension funds and life insurers, relating the investment manager's risk attitudes to the firm's long-term preferences is particularly important.

If investment opportunities are constant, the optimally designed benchmark aligns incentives perfectly. As such, optimally selecting the risk attitudes of investment managers becomes irrelevant. If investment opportunities are time-varying and we restrict attention to unconditional performance benchmarks, however, the benchmarks cannot overcome all inefficiencies and there is still scope for optimally selected asset managers. Table 3 assesses the value added by performance benchmarks once the risk attitudes of the asset managers are selected optimally. The results are provided for a range of risk attitudes of the CIO $\left(\gamma_{C}=4, \ldots, 10\right)$ and for horizons of $T_{C}=1$ and $T_{C}=10$. The first two columns report the costs of decentralized investment management in annualized terms for optimally selected asset managers. These costs range from 50 to 100 basis points per year for a CIO with a one-year investment horizon and from 200 to 400 basis points for an investment horizon of 10 years. Columns 3 and 4 show the costs if the CIO can optimally design unconditional benchmarks for each of the asset managers. Finally, the last two columns present the value of a benchmark in absolute terms and relative terms (in parentheses). The value of a benchmark 
ranges from 14 to 35 basis points for a $\mathrm{CIO}$ with a one year investment horizon, and from 22 to 47 basis points with a 10 year horizon. The relative improvement (as a fraction of total costs without a benchmark) varies from 10 to 30 percent.

We conclude that unconditional performance benchmarks are significantly value enhancing, even when risk attitudes of asset managers have been selected optimally. This extends the results of Admati and Pfleiderer (1997) concerning the role of performance benchmarks in delegated portfolio problems. In case of multiple asset managers, performance benchmarks can be useful in aligning incentives along, at least, three dimensions, namely diversification, preferences, and investment horizons. Moreover, we show quantitatively that the benchmark is rather effective in fulfilling this role.

\section{Extensions}

We extend the baseline results of sections 2 and 3 in several directions. First, managers of particular asset classes may be constrained from taking on short positions. We discuss the role of short sales constraints on the manager's portfolio choice and long-only benchmarks in Section 4.1. Second, asset managers are generally subjected to risk constraints, such as tracking error volatility constraints. Section 4.2 solves the problem under both absolute risk constraints and risk constraints relative to a benchmark. Third, institutional investors like pension funds and insurers need to account for liabilities in their asset allocation. Section 4.3 illustrates the impact of liabilities on the allocation of the CIO, the misalignments discussed above, and the role of optimal performance benchmarks in the presence of liabilities. For ease of exposition we assume that investment opportunities are constant.

\subsection{Short sales constraints}

Although institutional investors may be less restricted by short sales constraints than individuals, it is plausible that short-selling assets is costly for certain asset classes. In this section, we briefly summarize how long-only constraints can be incorporated.

In case of constant investment opportunities, Teplá (2000) shows that dynamic investment problems can be solved using standard static techniques. Specifically, in our setting, solving for the optimal portfolio of the asset managers entails solving a sequence of problems as in Section 2, but with a reduced asset set. Once the portfolio constraints are satisfied, we obtain a candidate solution and then optimize over all viable candidate solutions. When investment opportunities are time-varying, we have to resort to numerical techniques. However, numerical solutions are particularly simple to obtain because of the assumption of managerial 
myopia. Since the asset managers only care about current investment opportunities, we can solve for their optimal (constrained) allocations without solving a dynamic program.

Unfortunately, our empirical application is not particularly well-suited for imposing portfolio constraints, since the fixed income manager optimally shorts Aaa rated bonds to finance investments in Baa rated bonds and similarly for the equity manager for growth and value stocks. Consequently, we only obtain corner solutions.

\subsection{Risk constraints}

Apart from designing the optimal return benchmark, CIOs usually employ risk constraints in order to change or restrict the behavior of asset managers. These risk constraints can either be formulated in terms of absolute risk, in absence of a benchmark, or in terms of relative risk, when the asset manager is remunerated relative to a benchmark. Absolute risk constraints restrict the total volatility of the portfolio return. Relative risk constraints are concerned with the volatility of the portfolio return in excess of the benchmark return, as in Roll (1992) and Jorion (2003). We assume that the volatility constraints have to be satisfied at every point in time. In modern investment management firms, risk management systems monitor the risk exposures of portfolio holdings frequently, which makes it plausible to presume that risk constraints have to be satisfied continuously.

The instantaneous volatility of the portfolio return (absolute risk) is given by:

$$
\sigma^{A}\left(x_{i}\right)=\sqrt{x_{i}^{\prime} \Sigma_{i} \Sigma_{i}^{\prime} x_{i}}
$$

The instantaneous volatility of the portfolio return in excess of the benchmark (relative risk), also called the tracking error volatility, is given by:

$$
\sigma^{R}\left(x_{i}\right)=\sqrt{\left(x_{i}-\beta_{i}\right)^{\prime} \Sigma_{i} \Sigma_{i}^{\prime}\left(x_{i}-\beta_{i}\right)} .
$$

Using these definitions for absolute and relative risk, we impose risk limits of the form:

$$
\sigma^{j}\left(x_{i}\right) \leq \phi_{i j}
$$

with $j=A, R$. To ensure that the optimization problem of the asset managers is welldefined, we assume that:

$$
\sigma^{A}\left(x_{i}^{M V}\right) \leq \phi_{i A}
$$


which states that the limit on absolute risk must exceed the volatility of the minimum variance portfolio. In case of relative risk constraints, we require that $\phi_{i R} \geq 0$, since we restrict attention to benchmarks that can be replicated by the managers. A relative risk limit of $\phi_{i R}=0$ implies that the asset manager has the mandate to exactly implement the benchmark portfolio. We focus on the effect of imposing either of the two risk constraints, but not both. ${ }^{11}$

Whenever the unconstrained portfolio choice in absence of a benchmark does not violate the absolute risk constraint, this portfolio remains optimal for manager $i$. However, once the absolute risk constraint is violated, Appendix $\mathrm{C}$ shows that the optimal portfolio is:

$$
x_{i}^{N B}\left(\xi_{i}\right)=\frac{1}{\gamma_{i}\left(1+\xi_{i}\right)} x_{i}+\left(1-\frac{x_{i}^{\prime} \iota}{\gamma_{i}\left(1+\xi_{i}\right)}\right) x_{i}^{M V},
$$

where $x_{i}$ and $x^{M V}$ are given by equation (9) and $\xi_{i}>0$ satisfies:

$$
\sigma^{A}\left(x_{i}^{N B}\left(\xi_{i}\right)\right)=\phi_{A i}
$$

This solution shows that the absolute risk constraint induces an effective increase in relative risk aversion. Using the results of Section 2.5, it is easy to show that absolute risk constraints can mitigate inefficiencies whenever the investment manager is too aggressive. On the contrary, when the investment manager is too conservative, absolute risk constraints can actually aggravate the inefficiencies illustrated in Figure 3.

We also show in Appendix $\mathrm{C}$ that the optimal portfolio in the presence of a performance benchmark and binding relative risk constraints is given by:

$$
x_{i}^{B}\left(\xi_{i}\right)=\frac{1}{\gamma_{i}\left(1+\xi_{i}\right)} x_{i}+\left(1-\frac{1}{\gamma_{i}\left(1+\xi_{i}\right)}\right) \beta_{i}+\frac{1-x_{i}^{\prime} \iota}{\gamma_{i}\left(1+\xi_{i}\right)} x_{i}^{M V},
$$

where $x_{i}$ and $x^{M V}$ are given in equation (9) and $\xi_{i}>0$ satisfies:

$$
\sigma^{R}\left(x_{i}^{N B}\left(\xi_{i}\right)\right)=\phi_{R i}
$$

In addition, the appendix shows that the relative risk constraint binds for an investment manager with risk aversion $\gamma_{i}$ once the benchmark is designed on the basis of a higher risk aversion $\tilde{\gamma}_{i}$, with $\tilde{\gamma}_{i}>\gamma_{i}$. This implies that the CIO does not require specific knowledge of the manager's risk attitude more than knowing an upper bound. If the benchmark and relative risk constraint are designed on the basis of this conservative upper bound, the relative risk

\footnotetext{
${ }^{11}$ Jorion (2003) infers in addition the effect of both absolute and relative risk constraints.
} 
constraint binds for more aggressive managers. The binding constraint induces an effective increase in the manager's risk aversion to the level for which the benchmark is designed. Therefore, the results derived in Section 2 hold more broadly using relative risk constraints and require limited knowledge of the risk preferences of the asset managers.

In order to illustrate empirically the interplay between the managers' preferences and both absolute and relative risk, we provide in Figure 13 absolute and relative risks of the fixed income and stock managers when their relative risk aversion coefficients range from two to 10. The top graphs plot the absolute volatilities in absence of a benchmark for the fixed income manager (left) and the stock manager (right). The volatilities are monotonically decreasing in the risk aversion of the managers and are bounded below by the volatility of the minimum variance portfolios, with the minimum variance portfolio for stocks obviously being higher than that for bonds. The bottom graphs depict the relative risks (downwards sloping curves) and the corresponding absolute risks of the optimal portfolio in the presence of a benchmark. The relative risks are high for aggressive managers, but sharply decrease as the managers become more conservative. This holds for both asset classes. The absolute risk of the portfolios is independent of the risk preferences of the manager because the benchmark ensures that the manager implements the CIO's optimal relative portfolio.

\subsection{Liabilities}

When an investment management firm needs to meet future liabilities, decentralized investment management may give rise to yet another misalignment of incentives. Conventional benchmarks do not reflect the risks to which liabilities are exposed, and the presence of liabilities has therefore no effect on the portfolios of the asset managers. In this section, we quantify the inefficiencies induced by decentralized investment management in the presence of liabilities and discuss the design of liabilities-adjusted benchmarks.

We denote the value of the liabilities at time $t$ by $L_{t}$ and assume that the dynamics of the liabilities are given by:

$$
\frac{\mathrm{d} L_{t}}{L_{t}}=\mu_{L} \mathrm{~d} t+\sigma_{L}^{\prime} \mathrm{d} Z_{t}
$$

where $Z$ may contain an additional orthogonal Brownian motion to represent the risk in the liabilities that cannot be hedged. In that case, we add a column of zeros to the volatility matrix of the assets, $\Sigma$. The preferences of the CIO are modified to reflect the presence of the liabilities. In supervising pension funds and life insurers, it is common practice to measure the financial state of the fund by the funding ratio, which is the ratio of assets to 
liabilities. We therefore model the problem of the CIO in the presence of liabilities as:

$$
\max _{\left(x_{C s}\right)_{s \in\left[t, T_{C}\right]}} \mathbb{E}_{t}\left(\frac{1}{1-\gamma_{C}}\left(\frac{W_{T}}{L_{T}}\right)^{1-\gamma_{C}}\right)
$$

Using the results of Section 2.4, it is straightforward to show that the optimal portfolio of the $\mathrm{CIO}, x_{C}^{L}$, in case of constant investment opportunities is:

$$
x_{C}^{L}=x_{C}+\left(1-\frac{1}{\gamma_{C}}\right)\left(\Sigma \Sigma^{\prime}\right)^{-1} \Sigma \sigma_{L},
$$

where $x_{C}$ is given in equation (5) and the remainder, $1-x_{C}^{L \prime} \iota$, is invested in the cash account. Hence, in the presence of liabilities, the optimal portfolio contains a hedging component to hedge shocks that affect the value of the liabilities. The corresponding value function is:

$$
J\left(W / L, \tau_{C}\right)=\frac{1}{1-\gamma_{C}}\left(\frac{W}{L}\right)^{1-\gamma_{C}} \exp \left(a(x) \tau_{C}\right)
$$

with $\tau_{C}=T_{C}-t$ and

$$
\begin{aligned}
a(x)= & \left(1-\gamma_{C}\right)\left(r+x^{\prime} \Sigma \Lambda-\mu_{L}+\sigma_{L}^{\prime} \sigma_{L}-x^{\prime} \Sigma \sigma_{L}\right) \\
& -\frac{1}{2} \gamma_{C}\left(1-\gamma_{C}\right)\left(x^{\prime} \Sigma-\sigma_{L}^{\prime}\right)\left(\Sigma^{\prime} x-\sigma_{L}\right),
\end{aligned}
$$

where $x$ is the (implied) portfolio choice of the CIO. Since the asset managers are not directly affected by the liabilities, their optimal portfolios are unaltered. Hence, all results of Section 2 apply in the presence of liabilities, except that the optimal portfolio of the CIO is different. As the optimal benchmark is designed to implement the CIO's allocation in Section 2.4, the composition of the benchmark is influenced by the presence of the liabilities.

In order to assess empirically the impact of liabilities, we assume that the dynamics of the liabilities equal those of the government bonds, i.e. $\sigma_{L}=\Sigma(1,:)^{\prime}$. Correspondingly, the drift of the liabilities is given by $r+\sigma_{L}^{\prime} \Lambda$. The resulting portfolio of the CIO and, consequently, the performance benchmarks are tilted towards government bonds. In absence of a performance benchmark, Figure 14 illustrates the utility costs of decentralized investment management in the presence of liabilities. The numbers are strikingly higher than when the investment management firm does not have to account for liabilities. With the CIO's risk aversion equal to $\gamma_{C}=5$ in Panel A of Figure 14, the costs are in the order of three to four percent per year. For a more conservative $\mathrm{CIO}$ with $\gamma_{C}=10$ in Panel B of Figure 14, these costs range from five to seven percent per year. Therefore, the inefficiencies resulting from decentralized 
investment management are particularly pronounced in the presence of liabilities.

\section{Conclusions}

We address several misalignments of incentives induced by decentralized investment management. These misalignments between a CIO and the asset managers he employs can lead to large utility costs. One straightforward solution is to implement centralized investment strategies where the $\mathrm{CIO}$ attempts to manage all assets himself. However, from an organizational point of view, decentralized investment management is an inevitable and stylized fact of the investment industry. We show in this paper that the optimal design of an unconditional linear benchmark can be very effective in mitigating the costs of decentralized investment management. Furthermore, the optimal selection of the risk tolerances of the asset managers can lead to (further) reduction of these costs.

For ease of exposition, we confine attention to CRRA preferences and linear performance benchmarks. Future work could focus on a more complicated preference structure and/or non-standard contracts. For example, it seems reasonable that the utility function of the CIO is kinked as in van Binsbergen and Brandt (2005). The compensation scheme for the asset managers may also be non-linear and/or asymmetric, as in Browne (1999, 2000), Carpenter (2000), and Basak, Pavlova, and Shapiro (2005), for example. Another interesting extension is to assess the asset pricing implications of decentralized investment management. In delegated portfolio choice problems, Brennan (1993), Gómez and Zapatero (2003), and Cuoco and Kaniel (2003), illustrate the impact of delegation and benchmarking on equilibrium asset prices. Stutzer (2003b) shows that multiple benchmarks imply a factor model with these benchmarks returns as, possibly priced, factors. 


\section{References}

Admati, A.R., and P. Pfleiderer, 1997, Does it all add up? Benchmarks and the compensation of active portfolio managers, Journal of Business 70, 323-350.

Aït-Sahalia, Y., and M.W. Brandt, 2001, Variable selection for portfolio choice, Journal of Finance 56, 1297-1350.

Ang, A., and G. Bekaert, 2005, Stock return predictability, is it there?, working paper, Columbia University.

Ang, A., and J. Liu, 2004, How to discount cash-flows with time-varying expected returns, Journal of Finance 59, 2745-2783.

Barberis, N., 2000, Investing for the long run when returns are predictable, Journal of Finance 55, 225-264.

Barry, C.B., and L.T. Starks, 1984, Investment management and risk sharing with multiple managers, Journal of Finance 39, 477-491.

Basak, S., A. Pavlova, and A. Shapiro, Offsetting the incentives: Risk shifting and benefits of benchmarking in money management, working paper, London Business School.

Basak, S., A. Shapiro, and L. Teplá, 2005, Risk management with benchmarking, Management Science, forthcoming.

van Binsbergen, J.H., and M.W. Brandt, 2005, Optimal asset allocation in asset liability management, working paper, Duke University.

Brandt, M.W., 1999, Estimating portfolio and consumption choice: A conditional Euler equations approach, Journal of Finance 54, 1609-1645.

Brandt, M.W., 2005, Portfolio choice problems, in Y. Ait-Sahalia and L.P. Hansen (eds.), Handbook of Financial Econometrics, forthcoming.

Brennan, M.J., 1993, Agency and asset pricing, working paper, UCLA.

Brennan, M.J., E.S. Schwartz, and R. Lagnado, 1997, Strategic asset allocation, Journal of Economic Dynamics \& Control 21, 1377-1403.

Browne, S., 1999, Beating a moving target: Optimal portfolio strategies for outperforming a stochastic benchmark, Finance \& Stochastics 3, 275-294.

Browne, S., 2000, Risk constrained dynamic active portfolio management, Management Science 46, 1188-1199.

Campbell, J.Y., Y.L. Chan, and L.M. Viceira, 2003, A multivariate model of strategic asset allocation, Journal of Financial Economics 67, 41-80. 
Campbell, J.Y., and M. Yogo, 2005, Efficient tests of stock return predictability, Journal of Financial Economics, forthcoming.

Campbell, J.Y., and L.M. Viceira, 1999, Consumption and portfolio decisions when expected returns are time varying, Quarterly Journal of Economics 114, 433-496.

Campbell, J.Y., and T. Vuolteenaho, 2004, Bad beta, good beta, American Economic Review 94, 1249-1275.

Carpenter, J.N., 2000, Does option compensation increase managerial risk appetite?, Journal of Finance 55, 2311-2332.

Cochrane, J.H., and M. Piazzesi, 2005, Bond risk premia, American Economic Review 95, 138-160.

Cornell, B., and R. Roll, 2005, A delegated-agent asset-pricing model, Financial Analysts Journal 61, 57-69.

Cuoco, D., and R. Kaniel, 2003, Equilibrium prices in the presence of delegated portfolio management, working paper, University of Pennsylvania.

Cvitanić, J., A. Lazrak, L. Martellini, and F. Zapatero, 2005, Dynamic portfolio choice with parameter uncertainty and the economic value of analysts' recommendations, Review of Financial Studies, forthcoming.

Dai, Q., and K.J. Singleton, 2002, Expectation puzzles, time-varying risk premia, and affine models of the term structure, Journal of Financial Economics 63, 415-441.

Dessein, W., L. Garicano, and R. Gertner, 2005, Organizing for synergies, working paper, University of Chicago.

Elton, E.J., and M.J. Gruber, Optimum centralized portfolio construction with decentralized portfolio management, Journal of Financial and Quantitative Analysis 39, 481-494.

Foster, F.D., and M. Stutzer, 2003, Performance and risk aversion of funds with benchmarks: A large deviations approach, working paper, Australian Graduate School of Management.

Gómez, J.-P., and F. Zapatero, 2003, Asset pricing implications of benchmarking: A twofactor CAPM, European Journal of Finance 9, 343-357.

Jorion, P., 2003, Portfolio optimization with tracking-error constraints, Financial Analysts Journal 59, 70-82.

Jurek, J.W., and L.M. Viceira, 2005, Optimal Value and growth tilts in long-horizon portfolios, working paper, Harvard University.

Kim, T.S., and E. Omberg, 1996, Dynamic nonmyopic portfolio behavior, Review of Financial Studies 9, 141-162. 
Lewellen, J., 2004, Predicting returns with financial ratios, Journal of Financial Economics 74, 209-235.

Liu, J., 2006, Portfolio choice in stochastic environments, Review of Financial Studies, forthcoming.

Merton, R.C., 1969, Lifetime portfolio selection under uncertainty: The continuous-time case, Review of Economics and Statistics 51, 307-318

Merton, R.C., 1971, Optimum consumption and portfolio rules in a continuous-time model, Journal of Economic Theory 3, 373-413

Ou-Yang, H., 2003, Optimal contracts in a continuous-time delegated portfolio management problem, Review of Financial Studies 16, 173-208.

Roll, R., 1992, A mean/variance analysis of tracking rrror, Journal of Portfolio Management $18,13-22$.

Sangvinatsos, A., and J. Wachter, 2005, Does the failure of the expectations hypothesis matter for long-term investors?, Journal of Finance 60, 179-230.

Sharpe, W.F., 1981, Decentralized investment management, Journal of Finance 36, 217-234.

Stracca, L., 2005, Delegated portfolio management: A survey of the theoretical literature, working paper, European Central Bank.

Stutzer, M., 2003a, Portfolio choice with endogenous utility: A large deviations approach, Journal of Econometrics 116, 365-386.

Stutzer, M., 2003b, Fund managers may cause their benchmarks to be priced "risks", working paper, University of Colorado.

Teplá, L., 2000, Optimal portfolio policies with borrowing and shortsale constraints, Journal of Economic Dynamics \& Control 24, 1623-1639.

Treynor, J.L., and F. Black, 1973, How to use security analysis to improve portfolio selection, Journal of Business 46, 66-86.

Torous, W., R. Valkanov, and S. Yan, 2005, On predicting stock returns with nearly integrated explanantory variables, Journal of Business 77, 937-966.

Wachter, J.A., 2002, Portfolio and consumption decisions under mean-reverting returns: An exact solution for complete markets, Journal of Financial and Quantitative Analysis 37, 63-92. 


\section{A Constant investment opportunities}

\section{A.1 Centralized problem}

The centralized problem in Section 2.2 is the standard Merton $(1969,1971)$ problem. The dynamics of wealth are given by:

$$
\frac{\mathrm{d} W_{t}}{W_{t}}=\left(r+x_{C}^{\prime} \Sigma \Lambda\right) \mathrm{d} t+x_{C}^{\prime} \Sigma \mathrm{d} Z_{t} .
$$

The CIO can trade all $2 k$ assets and has access to a cash account. The Hamilton-JacobiBellman (HJB) equation is given by:

$$
\max _{x_{C}}\left(J_{W} W\left(x_{C}^{\prime} \Sigma \Lambda+r\right)+\frac{1}{2} W^{2} J_{W W} x_{C}^{\prime} \Sigma \Sigma^{\prime} x_{C}+J_{t}\right)=0
$$

where $J$ denotes the value function. The first-order condition (FOC) is:

$$
x_{C}=\frac{-J_{W}}{J_{W W} W}\left(\Sigma \Sigma^{\prime}\right)^{-1} \Sigma \Lambda .
$$

It is well-known that the value function takes the form:

$$
J\left(W, \tau_{C}\right)=\frac{1}{1-\gamma_{C}} W^{1-\gamma_{C}} \exp \left(a_{1} \tau_{C}\right),
$$

with $\tau_{C}=T_{C}-t$. Therefore, the composition of the optimal portfolio is given by:

$$
x_{C}=\frac{1}{\gamma_{C}}\left(\Sigma \Sigma^{\prime}\right)^{-1} \Sigma \Lambda,
$$

and the remainder, $1-x_{C}^{\prime} \iota$, is invested in the cash account. Substituting the optimal portfolio into the HJB equation provides the solution for $a_{1}$ :

$$
a_{1}=\left(1-\gamma_{C}\right) r+\frac{1}{2} \frac{\left(1-\gamma_{C}\right)}{\gamma_{C}} \Lambda^{\prime} \Sigma^{\prime}\left(\Sigma \Sigma^{\prime}\right)^{-1} \Sigma \Lambda
$$

\section{A.2 Decentralized problem without a benchmark}

In order to solve the decentralized problem in Section 2.3 without a performance benchmark, we first consider the problems of the two asset managers. Recall that both managers are cash constrained. The CIO then optimizes in the second step the strategic allocation to the two managers and allocates the remainder to the cash account.

Denote the manager's portfolio choice by $x_{i}^{N B}, i=1,2$. The wealth dynamics are:

$$
\frac{\mathrm{d} W_{t}}{W_{t}}=\left(r+x_{i}^{N{ }^{\prime \prime}} \Sigma_{i} \Lambda\right) \mathrm{d} t+x_{i}^{N{ }^{\prime \prime}} \Sigma_{i} \mathrm{~d} Z_{t},
$$

We drop the subscripts $i$ of the asset managers' wealth in the remainder of the appendices. 
The corresponding HJB equation is given by:

$$
\max _{x_{i}^{N B}: x_{i}^{N B^{\prime} \iota=1}}\left(J_{W} W\left(r+x_{i}^{N B^{\prime}} \Sigma_{i} \Lambda\right)+\frac{1}{2} W^{2} J_{W W} x_{i}^{N B^{\prime}} \Sigma_{i} \Sigma_{i}^{\prime} x_{i}^{N B}+J_{t}\right)=0 .
$$

The first order conditions of the Lagrangian corresponding to the (constrained) optimization problem are:

$$
\begin{aligned}
0 & =J_{W} W\left(r+\Sigma_{i} \Lambda\right)+W^{2} J_{W W} \Sigma_{i} \Sigma_{i}^{\prime} x_{i}^{N B}-\xi \iota \\
1 & =x_{i}^{N{ }^{\prime \prime} \iota},
\end{aligned}
$$

with $\xi$ denoting the Lagrange multiplier. The value function is of the form:

$$
J\left(W, \tau_{i}\right)=\frac{1}{1-\gamma_{i}} W^{1-\gamma_{i}} \exp \left(b \tau_{i}\right),
$$

with $\tau_{i}=T_{i}-t$. The solution to the FOCs provides the optimal portfolio choice of the asse managers given in equations (8) and (9).

In the second step, the CIO takes the portfolio choice of the portfolio managers as given. Therefore, the CIO solves an investment problem with two assets and a cash account, where the two assets have a volatility matrix:

$$
\bar{\Sigma}=\left[\begin{array}{c}
x_{1}^{N B^{\prime}} \Sigma_{1} \\
x_{2}^{N B^{\prime}} \Sigma_{2}
\end{array}\right]
$$

The results of Appendix A.1 directly apply, which yields the optimal portfolio allocations and value function given in equations (11)-(13).

\section{A.3 Decentralized problem with a benchmark}

We solve the decentralized problem with an optimally designed benchmark of Section 2.4. We derive first the optimal allocations of the asset managers in the presence of a benchmark. Define normalized wealth as $w_{i t}=W_{i t} B_{i t}^{-1}$. Recall that the benchmark comprises only positions in the assets available to the investment managers and no cash. The asset managers are therefore able to replicate the benchmark. The dynamics of the benchmark is given in equation (16). Using Ito's lemma, the dynamics of normalized wealth are:

$$
\frac{\mathrm{d} w_{t}}{w_{t}}=\left(x_{i}^{B \prime} \Sigma_{i} \Lambda-\beta_{i}^{\prime} \Sigma_{i} \Lambda+\beta_{i}^{\prime} \Sigma_{i} \Sigma_{i} \beta_{i}-\beta_{i}^{\prime} \Sigma_{i} \Sigma_{i}^{\prime} x_{i}^{B}\right) \mathrm{d} t+\left(x_{i}^{B \prime} \Sigma_{i}-\beta_{i}^{\prime} \Sigma_{i}\right) \mathrm{d} Z_{t} .
$$

The corresponding HJB equation is:

$$
\max _{x_{i}^{B}: x_{i}^{B^{\prime} \iota=1}}\left(\begin{array}{l}
J_{w} w\left(x_{i}^{B \prime} \Sigma_{i} \Lambda-\beta_{i}^{\prime} \Sigma_{i} \Lambda+\beta_{i}^{\prime} \Sigma_{i} \Sigma_{i} \beta_{i}-\beta_{i}^{\prime} \Sigma_{i} \Sigma_{i}^{\prime} x_{i}^{B}\right)+ \\
\frac{1}{2} w^{2} J_{w w}\left(x_{i}^{B \prime} \Sigma_{i}-\beta_{i}^{\prime} \Sigma_{i}\right)\left(x_{i}^{B \prime} \Sigma_{i}-\beta_{i}^{\prime} \Sigma_{i}\right)^{\prime}+J_{t}
\end{array}\right)=0 .
$$


The FOCs are:

$$
\begin{aligned}
0 & =J_{w} w\left(\Sigma_{i} \Lambda-\Sigma_{i} \Sigma_{i}^{\prime} \beta_{i}\right)+J_{w w} w^{2} \Sigma_{i}\left(\Sigma_{i}^{\prime} x_{i}^{B}-\Sigma_{i}^{\prime} \beta_{i}\right)-\xi \iota \\
1 & =x_{i}^{B \prime} \iota
\end{aligned}
$$

with $\xi$ denoting the Lagrange multiplier. The value function is of the form:

$$
J\left(W / B, \tau_{i}\right)=\frac{1}{1-\gamma_{i}}\left(\frac{W}{B}\right)^{1-\gamma_{i}} \exp \left(c \tau_{i}\right),
$$

with $\tau_{i}=T_{i}-t$. The solution of the FOCs is given by equation (18).

The CIO has to design the benchmarks, i.e. $\beta_{i}, i=1,2$, and decide on the strategic allocation to the managers and to the cash account. Since the managers' optimal portfolios are affine in the benchmark weights, see equation (18), the benchmark can be designed to solve for the optimal relative fractions invested in the different assets present in the asset classes. The strategic allocation, $x_{C} \in \mathbb{R}^{2}$, can subsequently be used to optimally manage the absolute fractions allocated to the different assets. More formally, in Appendix A.1, we show that when the CIO manages all assets individually, the optimal portfolio is:

$$
\begin{aligned}
x_{C} & =\frac{1}{\gamma_{C}}\left(\Sigma \Sigma^{\prime}\right)^{-1} \Sigma \Lambda \\
& =\left[\begin{array}{l}
x_{1 C} \\
x_{2 C}
\end{array}\right],
\end{aligned}
$$

where $x_{i C}$ denotes the allocation to the assets managed by manager $i$. We use $\beta_{i}$ to solve for the optimal relative fractions invested within the asset class:

$$
x_{i}^{B}=x_{i C}\left(x_{i C}^{\prime} \iota\right)^{-1} .
$$

Therefore, the optimal benchmark weights are given by:

$$
\beta_{i}=\frac{\gamma_{i}}{\gamma_{i}-1}\left[x_{i}^{C}\left(x_{i}^{C \prime} \iota\right)^{-1}-\left(\frac{1}{\gamma_{i}} x_{i}+\frac{1}{\gamma_{i}}\left(1-x_{i}^{\prime} \iota\right) x_{i}^{M V}\right)\right] .
$$

Note that $\beta_{i}^{\prime} \iota=1$, as required. Finally, the optimal allocation of the CIO's wealth to the managers is given by $x_{i C}^{\prime} \iota$. For this combination of benchmarks and the strategic allocation, the decentralized and centralized investment management problems coincide.

\section{A.4 Optimal investment manager selection}

We solve for the optimal risk attitudes of the investment managers and the allocation to the two managers. Define the risk tolerance as $\psi_{i}=\gamma_{i}^{-1}$. Given the optimal portfolio of investment managers without a benchmark in equation (8), the allocation to the different 
assets is given by:

$$
\left[\begin{array}{l}
x_{1 C}\left(\psi_{1}\left(x_{1}-\left(x_{1}^{\prime} \iota\right) x_{1}^{M V}\right)+x_{1}^{M V}\right) \\
x_{2 C}\left(\psi_{2}\left(x_{2}-\left(x_{2}^{\prime} \iota\right) x_{2}^{M V}\right)+x_{2}^{M V}\right)
\end{array}\right]
$$

which we equate to the optimal portfolio of the CIO when he can manage all four assets, the two tangency portfolios as well as the two minimum variance portfolios, independently. This results in the solution provided in Section 2.5.

\section{B Time-varying investment opportunities}

\section{B.1 Centralized problem}

The centralized problem in Section 3.2 relates to the portfolio choice problems in Sangvinatsos and Wachter (2005) and Liu (2006). The problem is solved using standard dynamic programming techniques. The joint dynamics of the state variables, $X$, is summarized by:

$$
\mathrm{d} X_{t}=K\left(\theta-X_{t}\right) \mathrm{d} t+\Sigma_{X} \mathrm{~d} Z_{t}
$$

and we assume in the text that $K$ is diagonal and $\theta=0_{m \times 1}$. The dynamics of wealth is:

$$
\frac{\mathrm{d} W_{t}}{W_{t}}=\left(r+x_{C}\left(X, \tau_{C}\right)^{\prime} \Sigma \Lambda(X)\right) \mathrm{d} t+x_{C}\left(X, \tau_{C}\right)^{\prime} \Sigma \mathrm{d} Z_{t}
$$

where $x_{C}\left(X, \tau_{C}\right)$ denotes the CIO's portfolio choice, conditional on both the state variables $(X)$ and the investment horizon $\left(\tau_{C}=T_{C}-t\right)$. The value function by $J_{1}$ depends on current wealth, the CIO's horizon, and, due to the Markovian structure of the financial market, on the current value of the state variables. The corresponding HJB equation is:

$$
\max _{x_{C}}\left(\begin{array}{c}
J_{W} W\left(r+x_{C}^{\prime} \Sigma \Lambda(X)\right)+\frac{1}{2} J_{W W} W^{2} x_{C}^{\prime} \Sigma \Sigma^{\prime} x_{C}+J_{t}+ \\
J_{X}^{\prime} K(\theta-X)+\frac{1}{2} \operatorname{tr}\left(\Sigma_{X}^{\prime} J_{X X} \Sigma_{X}\right)+W x_{C}^{\prime} \Sigma \Sigma_{X}^{\prime} J_{W X}
\end{array}\right)=0
$$

where we omit the indices of $x_{C}$ for notational convenience. The affine structure of the financial market implies that the value function is exponentially quadratic in the state variables:

$$
J\left(W, X, \tau_{C}\right)=\frac{W^{1-\gamma_{C}}}{1-\gamma_{C}} \exp \left(A\left(\tau_{C}\right)+B\left(\tau_{C}\right)^{\prime} X+\frac{1}{2} X^{\prime} C\left(\tau_{C}\right) X\right) .
$$

Solving for the FOC of provlem (B.3) and using equation (B.4) to determine the partial derivatives, we obtain:

$$
\begin{aligned}
x_{C}\left(X, \tau_{C}\right)= & \frac{1}{\gamma_{C}}\left(\Sigma \Sigma^{\prime}\right)^{-1} \Sigma \Lambda(X)+ \\
& \frac{1}{\gamma_{C}}\left(\Sigma \Sigma^{\prime}\right)^{-1} \Sigma \Sigma_{X}^{\prime}\left(B\left(\tau_{C}\right)+\frac{1}{2}\left(C\left(\tau_{C}\right)+C\left(\tau_{C}\right)^{\prime}\right) X\right) \\
= & \zeta_{0}^{C}\left(\tau_{C}\right)+\zeta_{1}^{C}\left(\tau_{C}\right) X,
\end{aligned}
$$


with

$$
\begin{aligned}
\zeta_{0}^{C}\left(\tau_{C}\right) & =\frac{1}{\gamma_{C}}\left(\Sigma \Sigma^{\prime}\right)^{-1} \Sigma \Lambda_{0}+\frac{1}{\gamma_{C}}\left(\Sigma \Sigma^{\prime}\right)^{-1} \Sigma \Sigma_{X}^{\prime} B\left(\tau_{C}\right), \\
\zeta_{1}^{C}\left(\tau_{C}\right) & =\frac{1}{\gamma_{C}}\left(\Sigma \Sigma^{\prime}\right)^{-1} \Sigma \Lambda_{1}+\frac{1}{2} \frac{1}{\gamma_{C}}\left(\Sigma \Sigma^{\prime}\right)^{-1} \Sigma \Sigma_{X}^{\prime}\left(C\left(\tau_{C}\right)+C\left(\tau_{C}\right)^{\prime}\right) .
\end{aligned}
$$

To find the coefficients $A, B$, and $C$, we substitute the optimal portfolio, equation (B.5), into the HJB equation (B.3) and match the constant, the terms linear in $X$, and the terms which are quadratic in $X$. In what follows, we derive the value function for any affine policy, $x(X, \tau)=\zeta_{0}(\tau)+\zeta_{1}(\tau) X$, which turns out be useful in subsequent derivations. The value function for this particular problem is obtained for $\zeta_{0}(\tau)=\zeta_{0}^{C}(\tau)$ and $\zeta_{1}(\tau)=\zeta_{1}^{C}(\tau)$. The resulting ODEs are: ${ }^{12}$

$$
\begin{aligned}
\dot{A}= & \left(1-\gamma_{C}\right)\left(r+\zeta_{0}^{\prime} \Sigma \Lambda_{0}\right)-\frac{1}{2} \gamma_{C}\left(1-\gamma_{C}\right) \zeta_{0}^{\prime} \Sigma \Sigma^{\prime} \zeta_{0}+B^{\prime} K \theta+ \\
& \frac{1}{4} \operatorname{tr}\left(\Sigma_{X}^{\prime}\left(C+C^{\prime}\right) \Sigma_{X}\right)+\frac{1}{2} B^{\prime} \Sigma_{X} \Sigma_{X}^{\prime} B+(1-\gamma) \zeta_{0}^{\prime} \Sigma \Sigma_{X}^{\prime} B \\
\dot{B}^{\prime}= & \left(1-\gamma_{C}\right)\left[\zeta_{0}^{\prime} \Sigma \Lambda_{1}+\Lambda_{0}^{\prime} \Sigma^{\prime} \zeta_{1}\right]-\gamma_{C}\left(1-\gamma_{C}\right) \zeta_{0}^{\prime} \Sigma \Sigma^{\prime} \zeta_{1}-B^{\prime} K+ \\
& \frac{1}{2} \theta^{\prime} K^{\prime}\left(C+C^{\prime}\right)+\frac{1}{2} B^{\prime} \Sigma_{X} \Sigma_{X}^{\prime}\left(C+C^{\prime}\right)+ \\
& \frac{1}{2}\left(1-\gamma_{C}\right) \zeta_{0}^{\prime} \Sigma \Sigma_{X}^{\prime}\left(C+C^{\prime}\right)+\left(1-\gamma_{C}\right) B^{\prime} \Sigma_{X} \Sigma^{\prime} \zeta_{1} \\
\dot{C}= & 2\left(1-\gamma_{C}\right) \zeta_{1}^{\prime} \Sigma \Lambda_{1}-\gamma_{C}\left(1-\gamma_{C}\right) \zeta_{1}^{\prime} \Sigma \Sigma^{\prime} \zeta_{1}-\left(C+C^{\prime}\right) K+ \\
& \frac{1}{4}\left(C+C^{\prime}\right) \Sigma_{X} \Sigma_{X}^{\prime}\left(C+C^{\prime}\right)+\left(1-\gamma_{C}\right) \zeta_{1}^{\prime} \Sigma \Sigma_{X}^{\prime}\left(C+C^{\prime}\right),
\end{aligned}
$$

subject to the boundary conditions:

$$
A(0)=0, B(0)=0_{k \times 1}, C(0)=0_{k \times k} .
$$

\section{B.2 Decentralized problem without a benchmark}

In the decentralized problem without a benchmark of Section 3.3, the asset managers implement active strategies. In order to solve for the myopic, cash constrained policy, the managers solve:

$$
\max _{x_{i}^{N B}: x_{i}^{N B^{\prime} \iota=1}}\left(\mathcal{L} u\left(W_{t}\right)\right),
$$

with $\mathcal{L}$ denoting the infinitesimal generator (which is equivalent to the differential generator in Merton (1971)). A straightforward application of Ito's lemma shows that this problem is equivalent to:

$$
\max _{x_{i}^{N B}: x_{i}^{N B^{\prime} \iota=1}} \mathbb{E}_{t}\left(x_{i}^{N B^{\prime}}(X) \Sigma_{i} \Lambda(X)-\frac{\gamma_{i}}{2} x_{i}^{N B^{\prime}}(X) \Sigma_{i} \Sigma_{i}^{\prime} x_{i}^{N B}(X)\right)
$$

\footnotetext{
${ }^{12}$ We suppress the time indexes of $\zeta_{0}$ and $\zeta_{1}$, as well as the coefficients of the conditional value function.
} 
As a result, the optimal strategy of the myopic, cash constrained investment managers is given by:

$$
\begin{aligned}
x_{i}^{N B}(X) & =\frac{1}{\gamma_{i}} x_{i}(X)+\left(1-\frac{x_{i}(X)^{\prime} \iota}{\gamma_{i}}\right) x_{i}^{M V} \\
& =\zeta_{0 i}^{N B}+\zeta_{1 i}^{N B} X,
\end{aligned}
$$

where $x_{i}(X)$ and $x_{i}^{M V}$ are given in equation (31) and:

$$
\begin{aligned}
\zeta_{0 i}^{N B} & =\frac{1}{\gamma_{i}}\left(\Sigma_{i} \Sigma_{i}^{\prime}\right)^{-1} \Sigma_{i} \Lambda_{0}+x_{i}^{M V}\left(1-\frac{\iota^{\prime}\left(\Sigma_{i} \Sigma_{i}^{\prime}\right)^{-1} \Sigma_{i} \Lambda_{0}}{\gamma_{i}}\right), \\
\zeta_{1 i}^{N B} & =\frac{1}{\gamma_{i}}\left(\Sigma_{i} \Sigma_{i}^{\prime}\right)^{-1} \Sigma_{i} \Lambda_{1}-x_{i}^{M V}\left(\frac{\iota^{\prime}\left(\Sigma_{i} \Sigma_{i}^{\prime}\right)^{-1} \Sigma_{i} \Lambda_{1}}{\gamma_{i}}\right) .
\end{aligned}
$$

These portfolio weights depend on the current value of the state variables and reflects, by construction, no horizon effects.

Anticipating the allocations of the asset managers, the CIO has to decide on the strategic allocation, i.e., how to allocate the funds available to the different managers. This strategic allocation is often fixed for a certain number of years and should reflect at least the firm's investment horizon. As such, we consider strategic allocations which are independent of the current state of the economy, but which do account for the investment horizon of the CIO. More specifically, we determine the optimal constant proportions strategy for the CIO. In order to solve for the optimal strategic allocation, we optimize the unconditional value function, i.e.:

$$
\mathbb{E}\left(J_{2}\left(W, X, \tau_{C}\right) \mid W\right)
$$

with

$$
J_{2}\left(W, X, \tau_{C}\right)=\mathbb{E}_{t}\left(\frac{1}{1-\gamma_{C}} W_{t+\tau_{C}}^{1-\gamma_{C}}\right)
$$

We first show that the conditional value function is exponentially affine in the state variables for any constant proportions strategic allocation. We then provide a lemma that can be used to determine the unconditional expectation of the conditional value function. Denote the allocation to the $i$ th asset manager by $x_{i C} \cdot{ }^{13}$ Given the optimal portfolios of the managers in (B.12), the resulting portfolio is given by:

$$
\begin{aligned}
x_{C}^{\text {Implied }} & =\left[\begin{array}{l}
x_{1 C}\left(\zeta_{01}^{N B}+\zeta_{11}^{N B} X\right) \\
x_{2 C}\left(\zeta_{02}^{N B}+\zeta_{12}^{N B} X\right)
\end{array}\right] \\
& =\zeta_{0}^{\text {Implied }}+\zeta_{1}^{\text {Implied }} X .
\end{aligned}
$$

Two aspects of the implied portfolio are worth noting. First, the implied portfolio is again affine in the state variables. Therefore, the conditional value function is of the form given in (B.4) with $\zeta_{0}(\tau)=\zeta_{0}^{\text {Implied }}$ and $\zeta_{1}(\tau)=\zeta_{1}^{\text {Implied }}$. We denote the value function in

\footnotetext{
${ }^{13} \mathrm{We}$ omit the indices of the portfolio strategies in this part of the derivation for notational convenience.
} 
absence of a benchmark by $J_{2}$. The ODEs required to determine the coefficients in the value function are given by equation (B.8). Second, the fact that the asset managers are assumed to time the market implies that the CIO has to allocate wealth to assets which exhibit a particular form of heteroskedasticity. Hence, although asset returns are homoskedastic in our model of the financial market, the timing ability of managers induces heteroskedastic asset returns at the CIO's level.

In order to determine the unconditional value function, we employ the following lemma proved by Ang and Liu (2004).

Lemma 1 Let $Y \in \mathbb{R}^{k \times 1}, Y \sim N(0, \Sigma), a \in \mathbb{R}^{k \times 1}$, and $B \in \mathbb{R}^{k \times k}$. If $\left(\Sigma^{-1}-2 B\right)$ is strictly positive definite, we have:

$$
\mathbb{E}\left(\exp \left(a^{\prime} Y+Y^{\prime} B Y\right)\right)=\exp \left(-\frac{1}{2} \ln \operatorname{det}(I-2 \Sigma B)+\frac{1}{2} a^{\prime}\left(\Sigma^{-1}-2 B\right)^{-1} a\right) .
$$

Using this lemma, it is straightforward to obtain the unconditional expectation of the conditional value function, $J_{2}$, which results in the unconditional value function. Next, we use numerical techniques to solve for the optimal strategic asset allocation, $x_{C}$. It is important to note that the coefficients in the conditional value function depend on the CIO's horizon. The strategic allocation will therefore be different for different investment horizons, i.e., $x_{C}\left(\tau_{C}\right)$.

\section{B.3 Decentralized problem with a benchmark}

In order to solve the decentralized investment problem of Section 3.4, in which a performance benchmark can be used to align incentives, we can largely use the results of Appendix B.2. The performance benchmark of manager $i$ is parameterized by a vector of constant portfolio weights, $\beta_{i}$, with the corresponding dynamics specified in equation (37). The asset manager is interested in wealth relative to the value of the benchmark. The dynamics of normalized wealth, $w_{i t}=W_{i t} B_{i t}^{-1}$, is given by:

$$
\begin{aligned}
\frac{\mathrm{d} w_{t}}{w_{t}}= & \left(x_{i}^{B \prime}(X) \Sigma_{i} \Lambda(X)-\beta_{i}^{\prime} \Sigma_{i} \Lambda(X)+\beta_{i}^{\prime} \Sigma_{i} \Sigma_{i} \beta_{i}-\beta_{i}^{\prime} \Sigma_{i} \Sigma_{i}^{\prime} x_{i}^{B}(X)\right) \mathrm{d} t+ \\
& \left(x_{i}^{B \prime}(X) \Sigma_{i}-\beta_{i}^{\prime} \Sigma_{i}\right) \mathrm{d} Z_{t} .
\end{aligned}
$$

where $x_{i}^{B}(X)$ denotes the myopic conditional portfolio choice of investment manager $i$. As in Appendix B.2 we set up the HJB equation, in which the maximization is subject to the condition that the managers have no access to a cash account, i.e., $x_{i}^{B \prime} \iota=1$. Solving for the FOCs and imposing the assumption of myopia, we obtain the optimal strategy implemented by the asset managers:

$$
\begin{aligned}
x_{i}^{B}(X) & =\frac{1}{\gamma_{i}} x_{i}(X)+\left(1-\frac{1}{\gamma_{i}}\right) \beta_{i}+\frac{1}{\gamma_{i}}\left(1-x_{i}(X)^{\prime} \iota\right) x_{i}^{M V} \\
& =\zeta_{0 i}^{B}+\zeta_{1 i}^{B} X
\end{aligned}
$$


with $x_{i}(X)$ and $x_{i}^{M V}$ as in equation (31) and:

$$
\begin{aligned}
\zeta_{0 i}^{B} & =\frac{1}{\gamma_{i}}\left(\Sigma_{i} \Sigma_{i}^{\prime}\right)^{-1} \Sigma_{i} \Lambda_{0}+\left(1-\frac{1}{\gamma_{i}}\right) \beta_{i}+\frac{1}{\gamma_{i}} x_{i}^{M V}\left(1-\iota^{\prime}\left(\Sigma_{i} \Sigma_{i}^{\prime}\right)^{-1} \Sigma_{i} \Lambda_{0}\right) \\
\zeta_{1 i}^{B} & =\frac{1}{\gamma_{i}}\left(\Sigma_{i} \Sigma_{i}^{\prime}\right)^{-1} \Sigma_{i} \Lambda_{1}-\frac{1}{\gamma_{i}} x_{i}^{M V}\left(\iota^{\prime}\left(\Sigma_{i} \Sigma_{i}^{\prime}\right)^{-1} \Sigma_{i} \Lambda_{1}\right)
\end{aligned}
$$

The implication of equation (B.20) is that the optimal portfolio of the managers is again affine in the state variables. Hence, we proceed as in Appendix B.2. The CIO selects the optimal constant proportions strategy and the constant benchmarks, $\beta_{1}$ and $\beta_{2}$, to optimize the unconditional value function, i.e. equation (B.15) and equation (B.16). This yields:

$$
\begin{aligned}
x_{C}^{\text {Implied }} & =\left[\begin{array}{l}
x_{1 C}\left(\zeta_{01}^{B}+\zeta_{11}^{B} X\right) \\
x_{2 C}\left(\zeta_{02}^{B}+\zeta_{12}^{B} X\right)
\end{array}\right] \\
& =\zeta_{0}^{\text {Implied }}+\zeta_{1}^{\text {Implied }} X,
\end{aligned}
$$

where $\zeta_{0 i}^{B}$ and $\zeta_{1 i}^{B}$ obviously depend on the choice of the benchmark. The conditional value function is exponentially quadratic as in equation (B.4), with $\zeta_{0}(\tau)=\zeta_{0}^{\text {Implied }}$ and $\zeta_{1}(\tau)=\zeta_{1}^{\text {Implied }}$. We denote the conditional value function in the presence of a performance benchmark by $J_{3}$. The coefficients satisfy the ODEs given in (B.8). To solve for the strategic allocation and the performance benchmark, we evaluate the unconditional expectation of the conditional value function using Lemma 1 . We then optimize numerically.

\section{B.4 Optimal selection of investment managers}

To solve for the optimal choice of asset managers, we use the results of Appendix B.2, where the unconditional value function is given in (B.15) as a function of the risk attitudes of the managers and the strategic allocation. Hence, the numerical optimization of the unconditional value function in equation (B.15) is performed over both the strategic allocation, $x_{C}$, and the risk attitudes of the asset managers, $\gamma_{1}$ and $\gamma_{2}$.

\section{Extensions}

\section{C.1 Risk constraints}

We derive in this section the optimal allocations of the asset managers in the presence of either relative or absolute risk constraints as defined in Section 4.2. We assume investment opportunities to be constant and asset managers to behave myopically. We maintain in addition the cash constraint, implying that the portfolio weights have to sum to one.

For the case with absolute risk constraints, asset manager $i$ solves:

$$
\max _{x_{i}^{N B} \in \mathcal{A}_{i}}\left(\mathcal{L} u\left(W_{t}\right)\right)
$$


with $\mathcal{L}$ denoting the infinitesimal generator and the set $\mathcal{A}_{i}$ is given by:

$$
\mathcal{A}_{i}=\left(x \mid x^{\prime} \iota=1, \sqrt{x^{\prime} \Sigma_{i} \Sigma_{i}^{\prime} x} \leq \phi_{A i}\right)
$$

The objective function in equation (C.1) is equivalent to:

$$
\max _{x_{i}^{N B} \in \mathcal{A}_{i}}\left(x_{i}^{N B^{\prime}} \Sigma_{i} \Lambda+r-\frac{\gamma}{2} x_{i}^{N B^{\prime}} \Sigma_{i} \Sigma_{i}^{\prime} x_{i}^{N B}\right) .
$$

Consequently, the Kuhn-Tucker FOCs are:

$$
\begin{aligned}
0 & =\Sigma_{i} \Lambda-\gamma\left(1+\xi_{1}\right) \Sigma_{i} \Sigma_{i}^{\prime} x_{i}^{N B}-\xi_{1} \iota \\
1 & =x_{i}^{N{ }^{\prime}} \iota, \phi_{A i}^{2} \geq x_{i}^{N{ }^{\prime}} \Sigma_{i} \Sigma_{i}^{\prime} x_{i}^{N B}, \xi_{2} \geq 0 \\
0 & =\xi_{2}\left(\phi_{A i}^{2}-x_{i}^{N B^{\prime}} \Sigma_{i} \Sigma_{i}^{\prime} x_{i}^{N B}\right)
\end{aligned}
$$

with $\xi_{1}$ and $\xi_{2}$ denoting the Kuhn-Tucker multipliers. ${ }^{14}$ If the risk constraint is not binding, the managers' optimal portfolio is as derived in Section 2.3. Otherwise, the absolute risk constraint binds and the optimal portfolio is given by the solution to equation (C.4) for $\xi_{2}>0$ so that the risk constraint holds with equality. This results immediately in the optimal portfolio given in equation (44).

When the asset managers have to satisfy relative risk constraints, their objective is:

$$
\max _{x_{i}^{B} \in \mathcal{B}_{i}}\left(\mathcal{L} u\left(\frac{W_{t}}{B_{t}}\right)\right),
$$

where the set $\mathcal{B}_{i}$ is given by:

$$
\mathcal{B}_{i}=\left(x \mid x^{\prime} \iota=1, \sqrt{\left(x-\beta_{i}\right)^{\prime} \Sigma_{i} \Sigma_{i}^{\prime}\left(x-\beta_{i}\right)} \leq \phi_{R i}\right) .
$$

Proceeding as above, the FOCs are:

$$
\begin{aligned}
0 & =\Sigma_{i}\left(\Lambda-\Sigma_{i} \beta_{i}\right)-\gamma\left(1+\xi_{1}\right) \Sigma_{i} \Sigma_{i}^{\prime}\left(x_{i}^{B}-\beta_{i}\right)-\xi_{1} \iota \\
1 & =x_{i}^{B \prime} \iota, \phi_{R i}^{2} \geq\left(x_{i}^{B}-\beta_{i}\right)^{\prime} \Sigma_{i} \Sigma_{i}^{\prime}\left(x_{i}^{B}-\beta_{i}\right), \xi_{2} \geq 0 \\
0 & =\xi_{2}\left(\phi_{R i}^{2}-\left(x_{i}^{B}-\beta_{i}\right)^{\prime} \Sigma_{i} \Sigma_{i}^{\prime}\left(x_{i}^{B}-\beta_{i}\right)\right)
\end{aligned}
$$

where $\xi_{1}$ and $\xi_{2}$ indicate the Kuhn-Tucker multipliers. Again, if the relative risk constraint is not binding, the optimal portfolio of Section 2.4 prevails. Otherwise, the optimal investment strategy of manager $i$ is given by the solution to equation (C.9) with $\xi_{2}>0$ so that the relative risk constraint is satisfied with equality. This readily implies the strategy given in equation (46).

Finally, we show that if the benchmark is designed on the basis of a higher risk aversion level, say $\tilde{\gamma}$, than the manager's risk aversion, denoted by $\gamma$, the relative risk of the manager's portfolio will exceed the risk of a manager with a risk aversion level $\tilde{\gamma}$. Hence, if the risk

\footnotetext{
${ }^{14} \mathrm{In}$ fact, $\xi_{2}$ is the multiplier for the risk constraint scaled by a factor $\gamma / 2$ to simplify the interpretation.
} 
limit is constructed as if the manager's risk aversion equals $\tilde{\gamma}$, the relative risk constraint will bind and induces an effective increase in the manager's risk aversion from $\gamma$ to $\tilde{\gamma}$. Towards this end, note that the difference between the optimal portfolio of the manager, who has a risk aversion $\gamma$, and the benchmark weights, which are designed for a manager with risk aversion $\tilde{\gamma}$, is given by:

$$
x^{B}(\gamma, \beta(\tilde{\gamma}))-\beta(\tilde{\gamma})=\frac{\tilde{\gamma}}{\tilde{\gamma}-1} \frac{1}{\gamma}\left\{x_{i}-x_{i}^{C}\left(x_{i}^{C \prime} \iota\right)^{-1}+\left(1-\iota^{\prime} x_{i}\right) x_{i}^{M V}\right\},
$$

where $x^{B}(\gamma, \beta(\tilde{\gamma}))$ denotes the optimal portfolio choice when the investor has a coefficient of relative risk aversion $\gamma$, but is evaluated relative to a benchmark, $\beta(\tilde{\gamma})$, which is based on $\tilde{\gamma}$. This immediately implies for the relative risk of the manager's portfolio:

$$
\begin{aligned}
\left(x^{B}(\gamma, \beta(\tilde{\gamma}))-\beta(\tilde{\gamma})\right)^{\prime} & \left(\Sigma_{i} \Sigma_{i}^{\prime}\right)\left(x^{B}(\gamma, \beta(\tilde{\gamma}))-\beta(\tilde{\gamma})\right)= \\
& \left(\frac{\tilde{\gamma}}{\gamma}\right)^{2}\left(x^{B}(\tilde{\gamma}, \beta(\tilde{\gamma}))-\beta(\tilde{\gamma})\right)^{\prime}\left(\Sigma_{i} \Sigma_{i}^{\prime}\right)\left(x^{B}(\tilde{\gamma}, \beta(\tilde{\gamma}))-\beta(\tilde{\gamma})\right),
\end{aligned}
$$

i.e. the relative risk of a more aggressive manager under a benchmark designed for a more conservative manager is larger than when the more conservative manager implements the strategy, since $\tilde{\gamma}>\gamma$. This implies that when the risk constraint is satisfied with equality for a manager with risk aversion $\tilde{\gamma}$, an unconstrained manager with risk aversion $\gamma$ will implement a strategy which exceeds the relative risk limit. Consequently, the risk constraint on the basis of which the benchmark is designed will be binding and induces an effective increase in the manager's risk aversion from $\gamma$ to $\tilde{\gamma}$. 


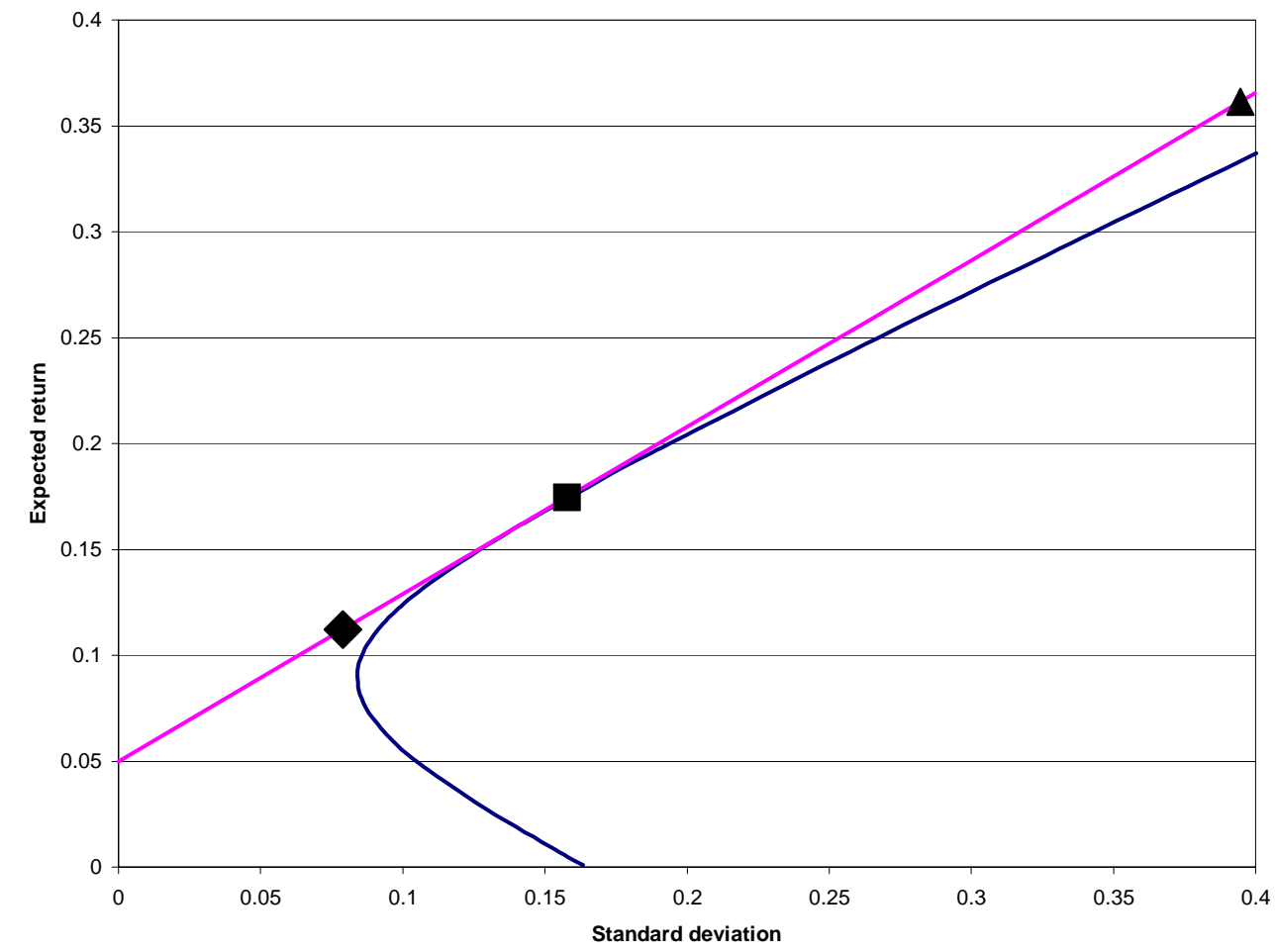

- Optimal MV frontier — Optimal Sharpe ratio $\Delta$ gamma $=2 \quad$ agamma $=5 \quad \bullet$ gamma $=10$

Figure 1: Mean-variance frontier in the centralized problem. Mean-variance frontier of a centralized asset allocation problem with government bonds, long-term corporate Baa bonds, long-term Aaa bonds, growth stocks, intermediate, and value stocks. The figure also indicates the portfolio choices of a CIO with risk aversion of respectively $\gamma_{C}=2,5$, or 10 . 


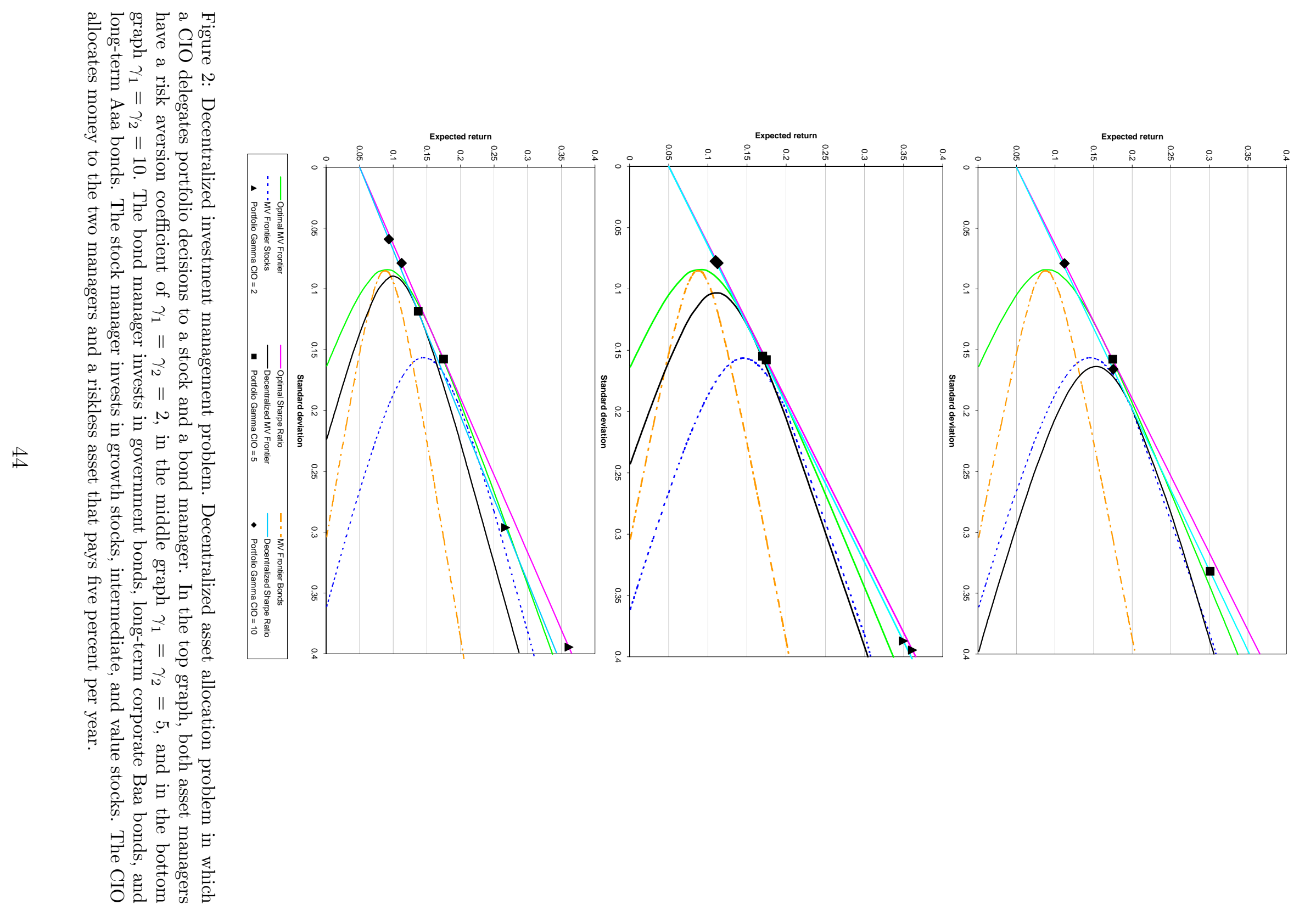


Panel A: The coefficient of relative risk aversion of the CIO equals $\gamma_{C}=5$

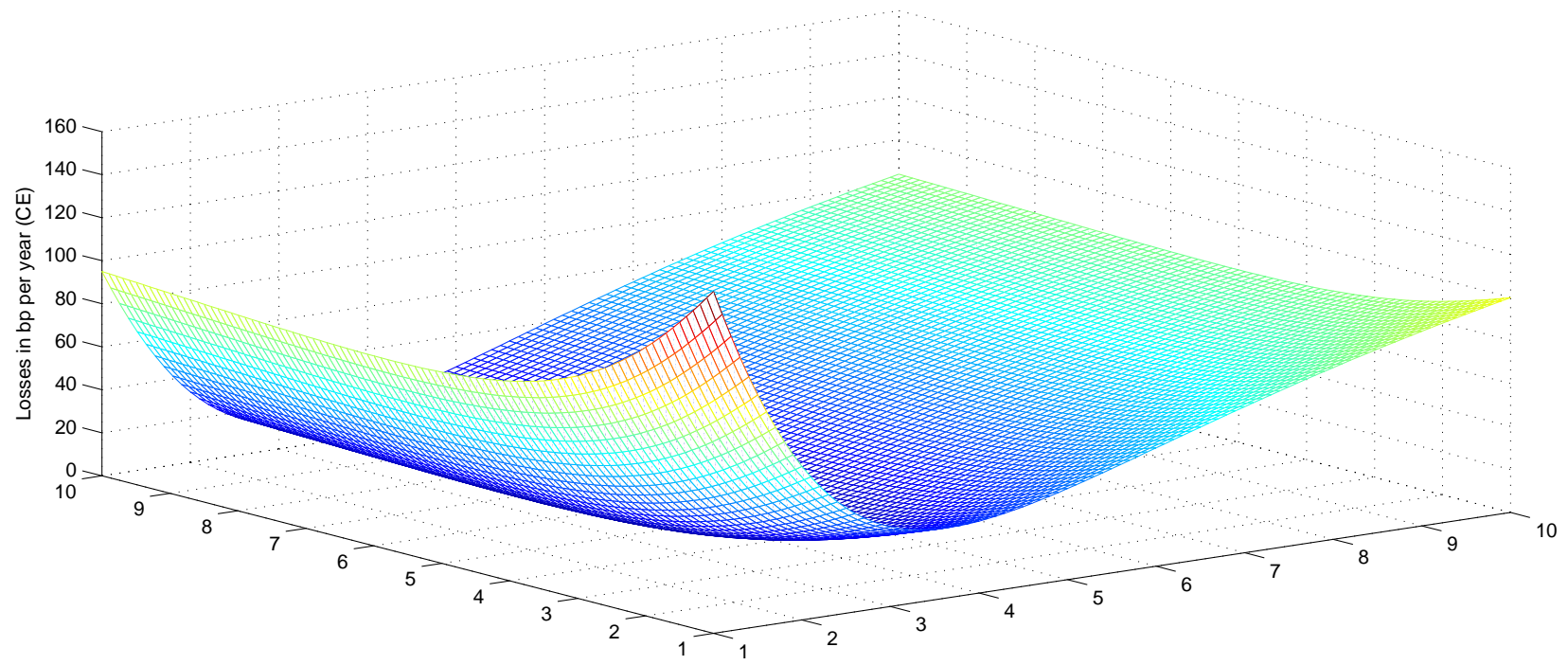

Risk aversion bond manager

Risk aversion stock manager

Panel B: The coefficient of relative risk aversion of the CIO equals $\gamma_{C}=10$

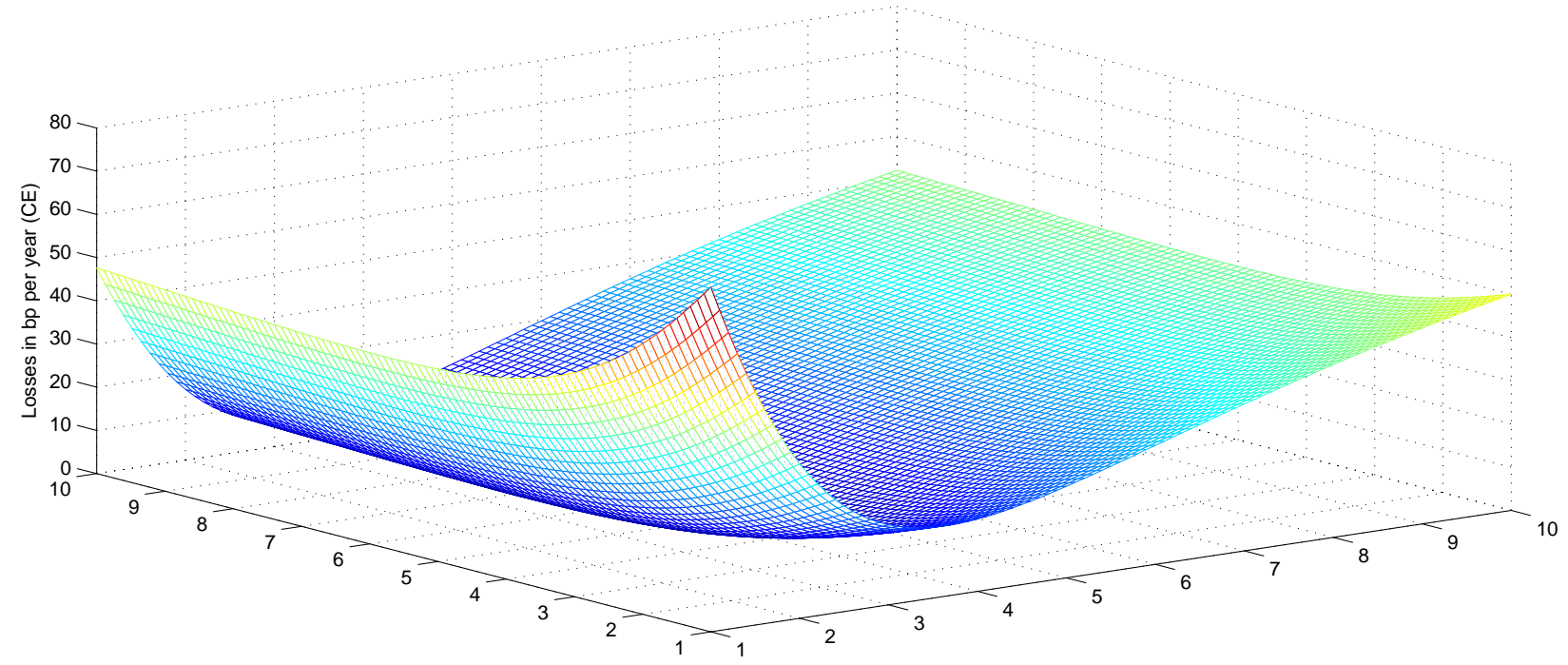

Risk aversion bond manager

Risk aversion stock manager

Figure 3: Losses from decentralized investment management in basis points per year (CE). Diversification losses due to decentralized investment management as a function of the risk aversion of the investment managers. The CIO has a risk aversion coefficient $\gamma_{C}=5$ in Panel A and $\gamma_{C}=10$ in Panel B. The losses are computed by taking the ratio of the certainty equivalents achieved under centralized and decentralized investment management after which we subtract one and multiply by -10,000 to express the losses in bp per year. For example 160 basis points implies a loss in terms of certainty equivalents of 1.6 percent of wealth per year. 
Panel A: Portfolio composition bond manager

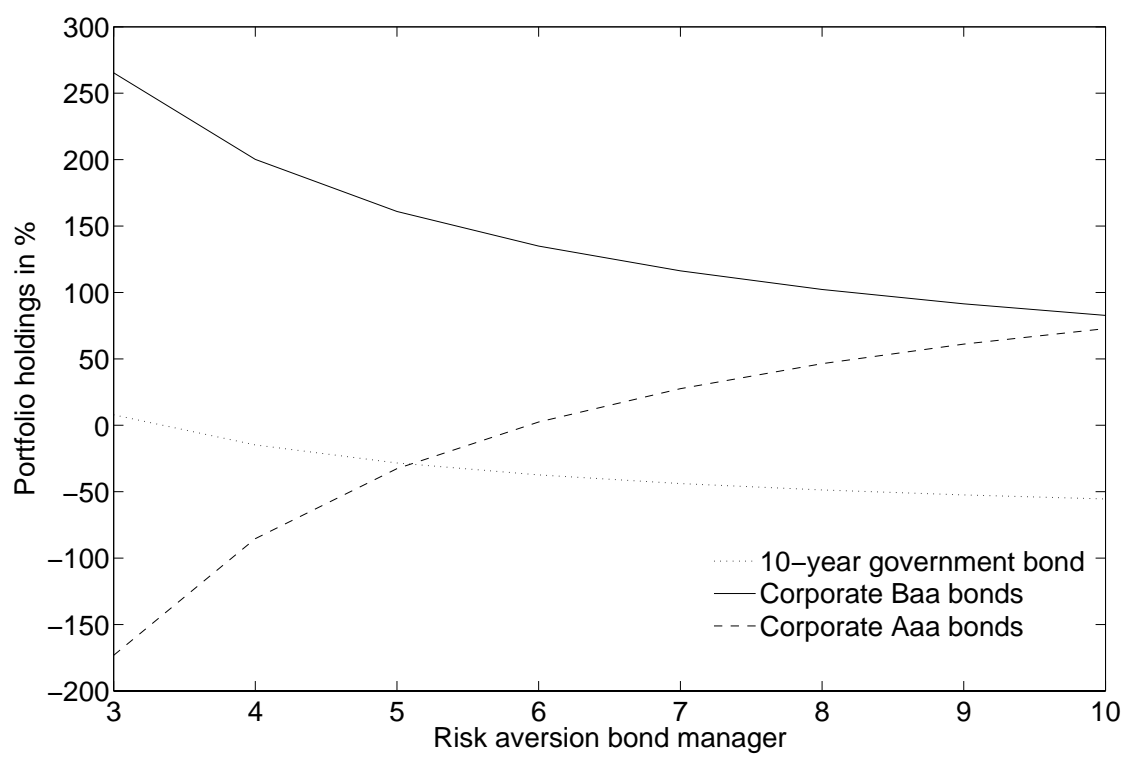

Panel B: Portfolio composition stock manager

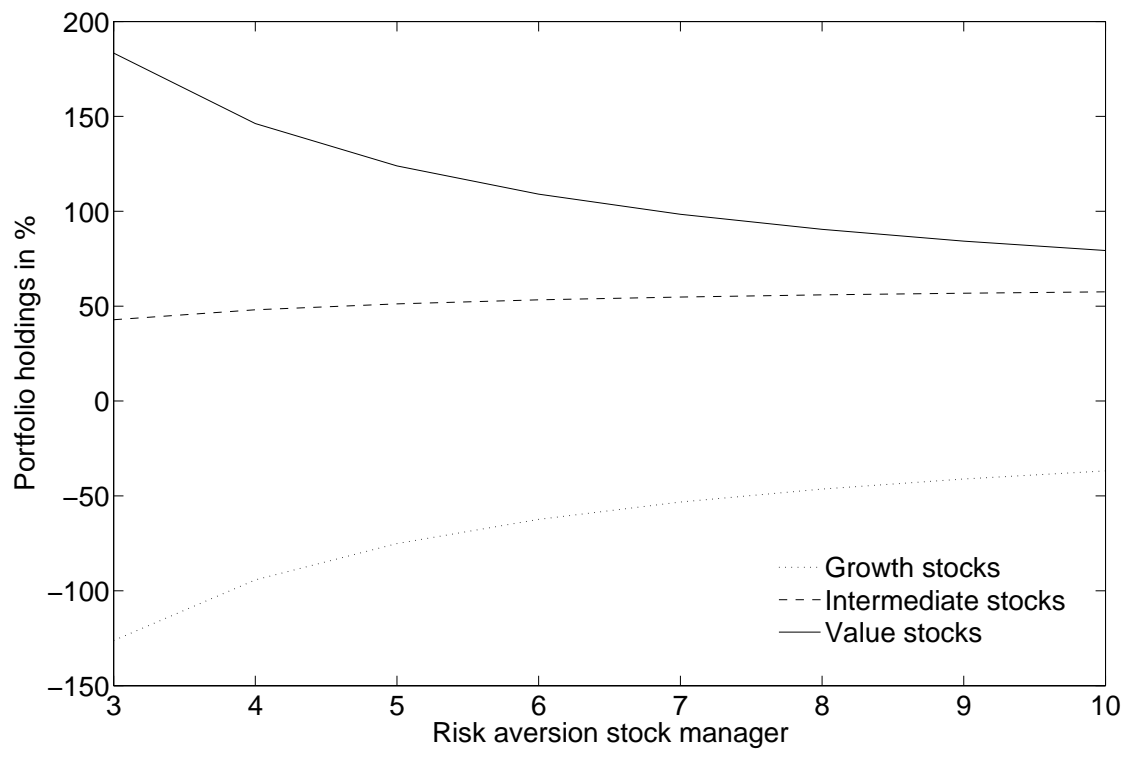

Figure 4: Portfolio compositions without a benchmark. Portfolio composition of the bond manager in Panel $\mathrm{A}$ and the stock manager in Panel B as a function of their coefficients of relative risk aversion when they are not restricted by a benchmark. The asset managers do not have access to a riskless asset. 


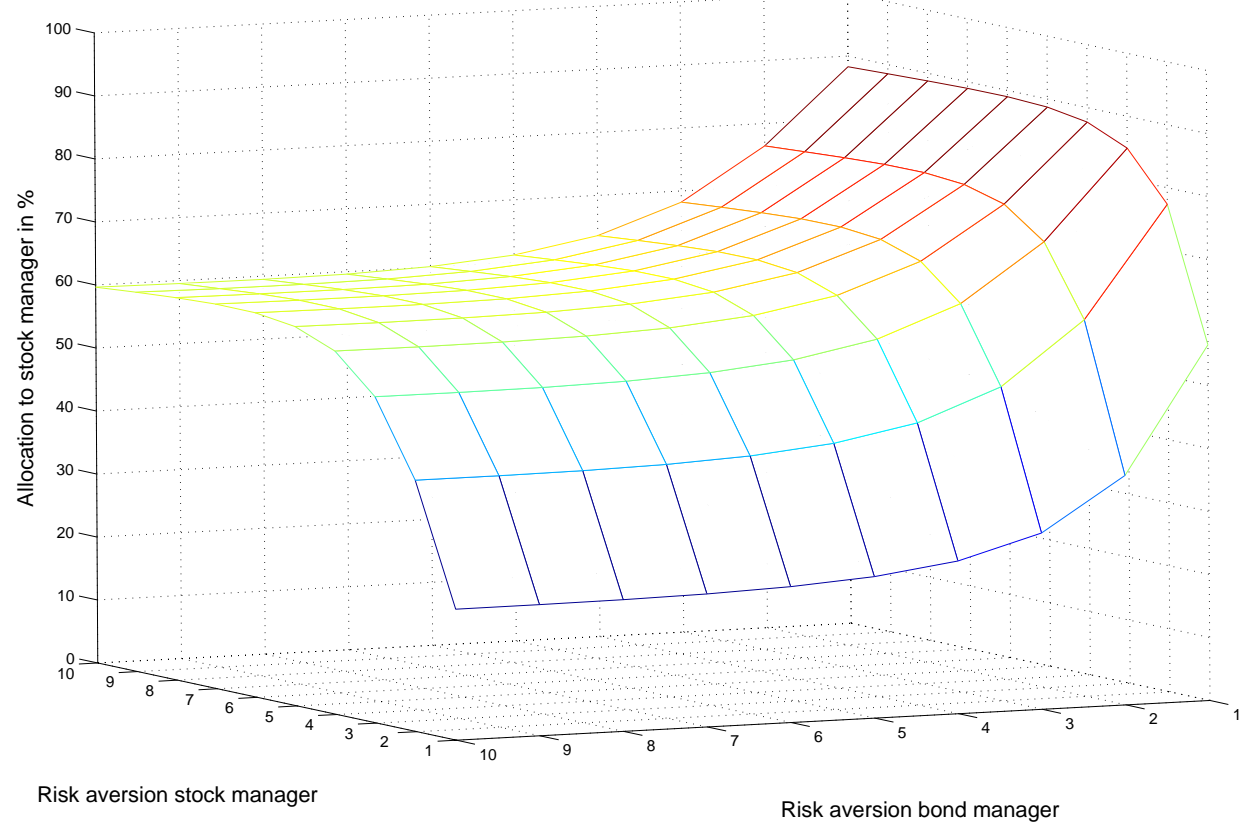

Figure 5: Fraction of risky funds allocated to the equities asset class if there is no benchmark. Percentage of total investment in risky assets that is under control of the stock manager as a function of the risk aversion of the bond and the stock manager. 
Panel A: Composition of the optimal bond benchmark

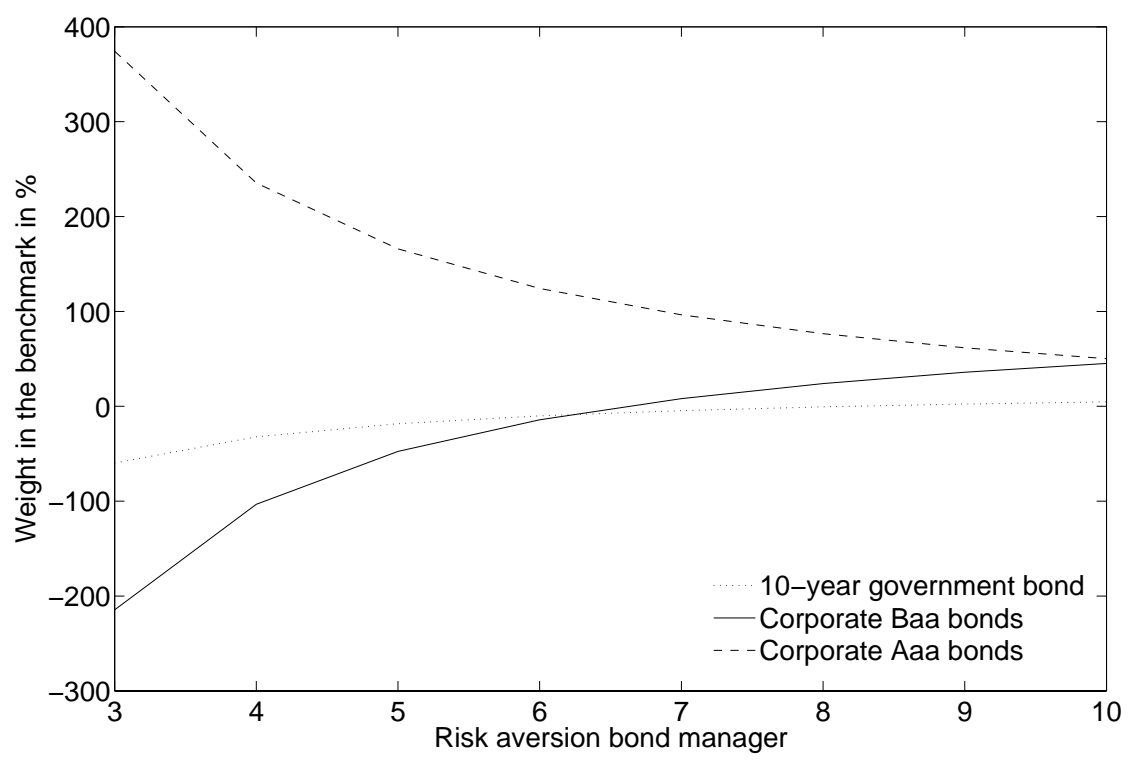

Panel B: Composition of the optimal stock benchmark

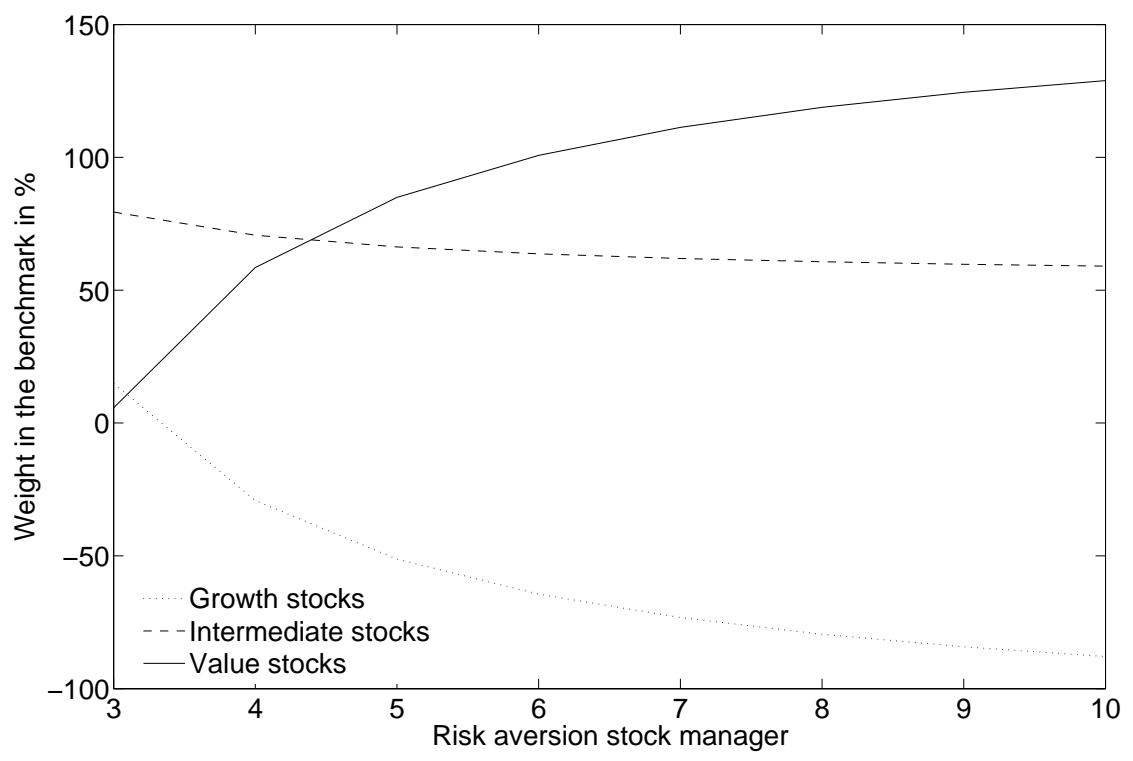

Figure 6: Composition of the optimal performance benchmarks. Composition of the optimal bond benchmark in Panel A and stock benchmark in Panel B as a function of the risk aversion of the asset managers. 
Panel A: The coefficient of relative risk aversion of the CIO equals $\gamma_{C}=5$

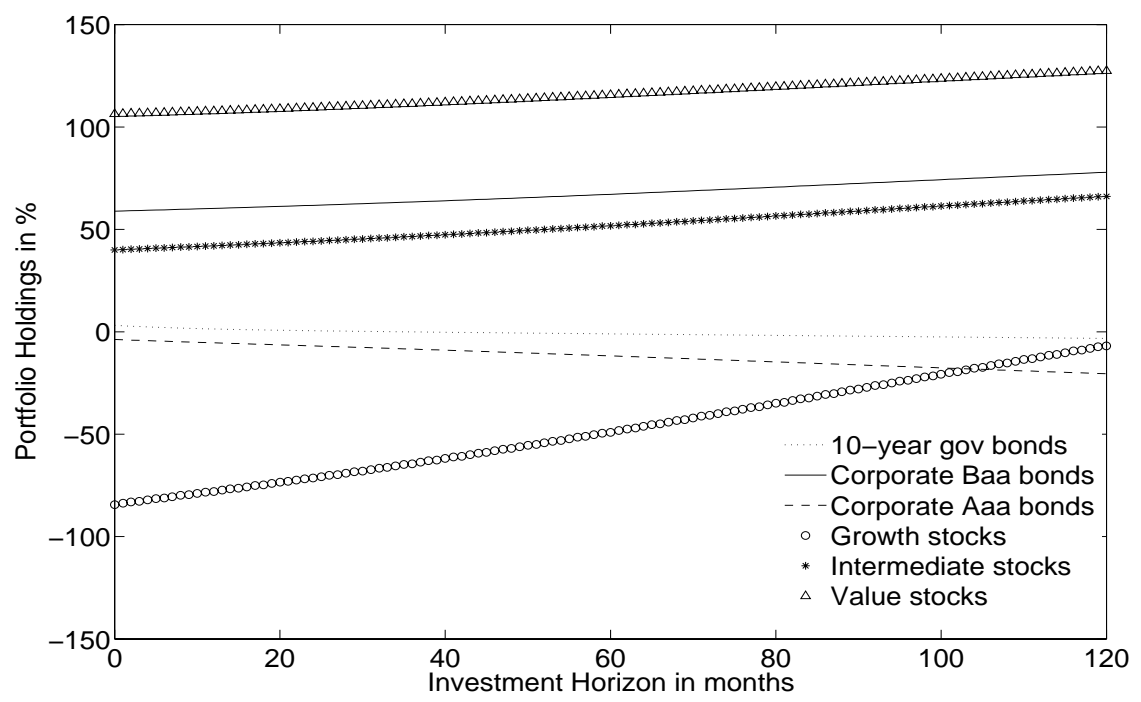

Panel B: The coefficient of relative risk aversion of the CIO equals $\gamma_{C}=10$

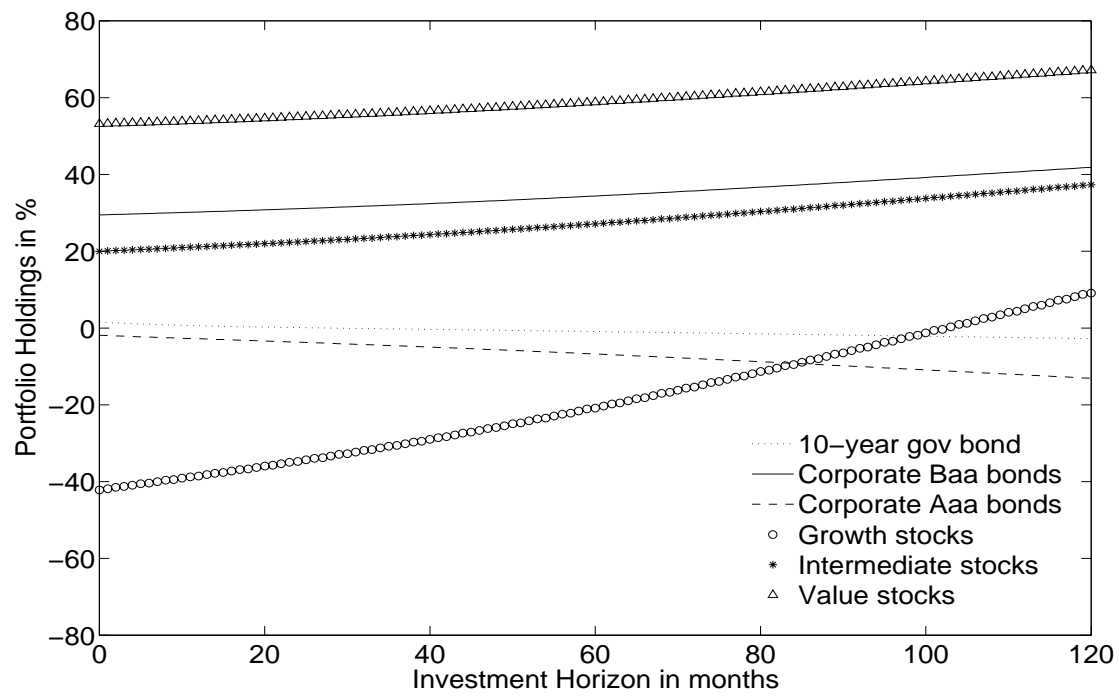

Figure 7: Optimal portfolio choice in the centralized problem. Optimal allocation to government bonds, corporate bonds with ratings Baa and Aaa, and three stock portfolios ranked on their book-to-market ratios (growth, intermediate, and value). The horizontal axis depicts the investment horizon of the CIO in months. The coefficient of relative risk aversion of the CIO equals $\gamma_{C}=5$ in Panel A and $\gamma_{C}=10$ in Panel B. 


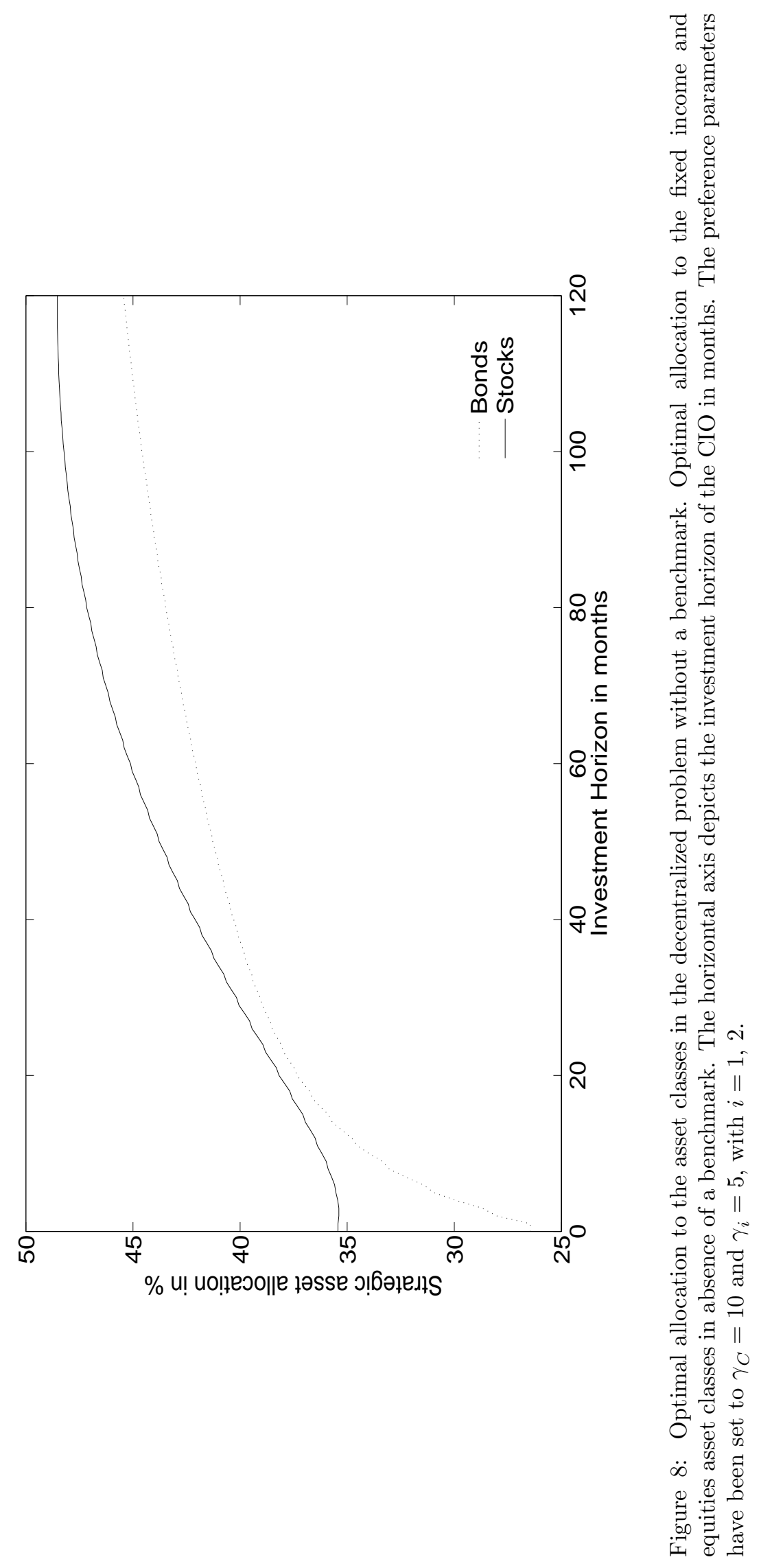


Panel A: The investment horizon of the CIO equals $T=1$

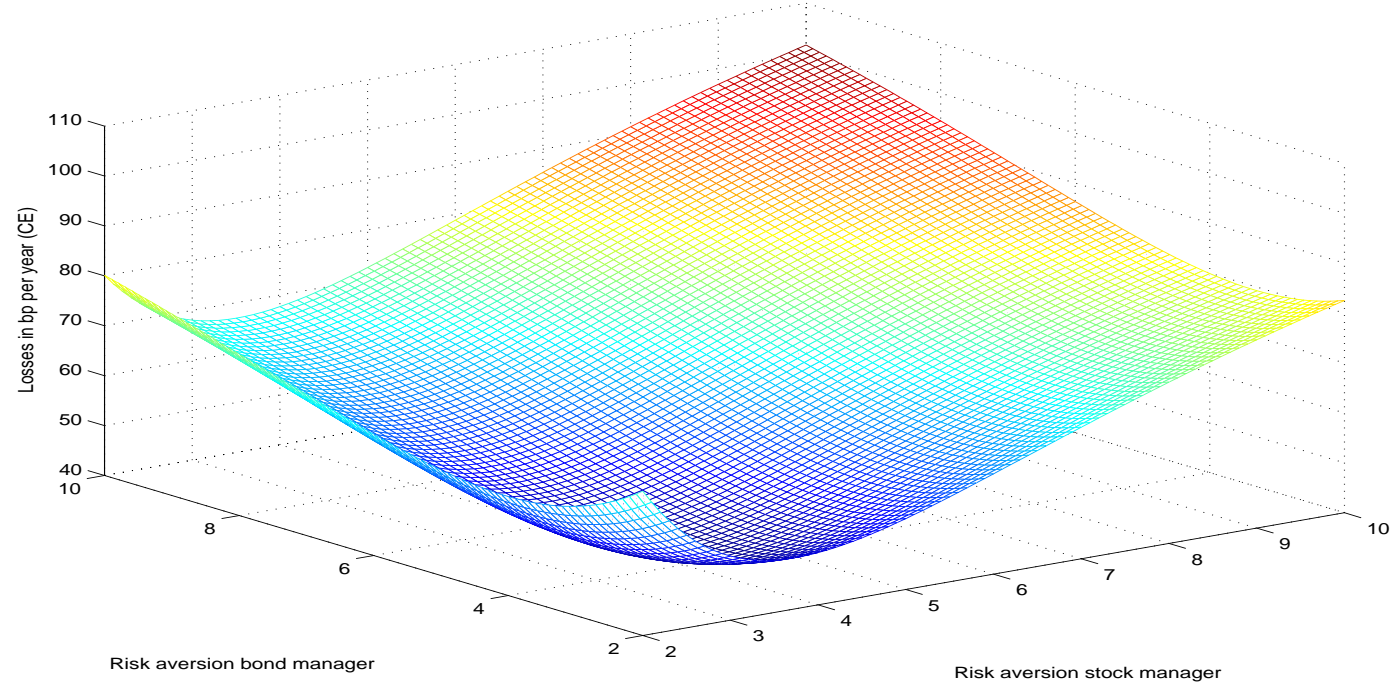

Panel B: The investment horizon of the CIO equals $T=10$

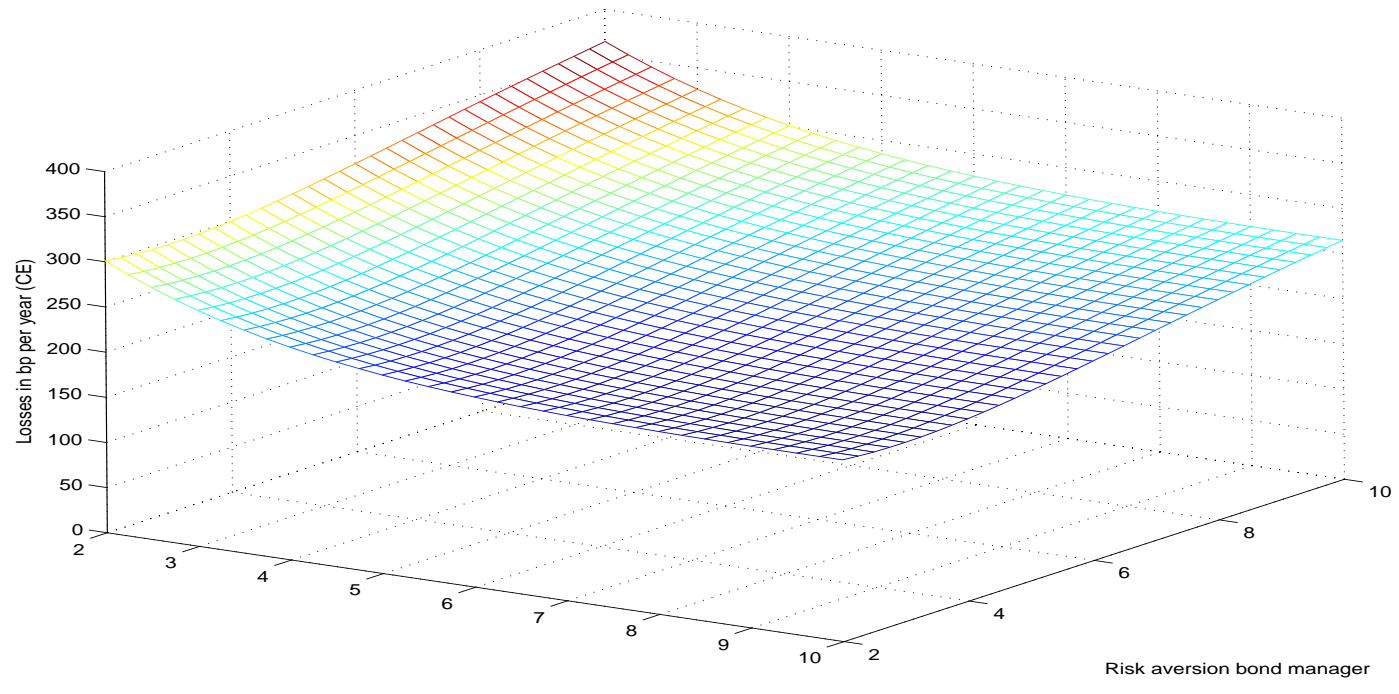

Risk aversion stock manager

Figure 9: Utility costs of decentralized investment management when there is no benchmark. Comparison of certainty equivalents following from the centralized and decentralized investment management problem when there is no benchmark and the investment horizon is one year in Panel A and 10 years in Panel B. The $\mathrm{x}$ - and $\mathrm{y}$-axis depict the risk attitudes of the asset managers. The coefficient of relative risk aversion of the CIO equals 10. The losses are computed by taking the ratio of the certainty equivalents achieved under centralized and decentralized investment management after which we subtract one and multiply by -10,000 to express the losses in basis points per year. 
Panel A: Composition of the optimal performance benchmarks

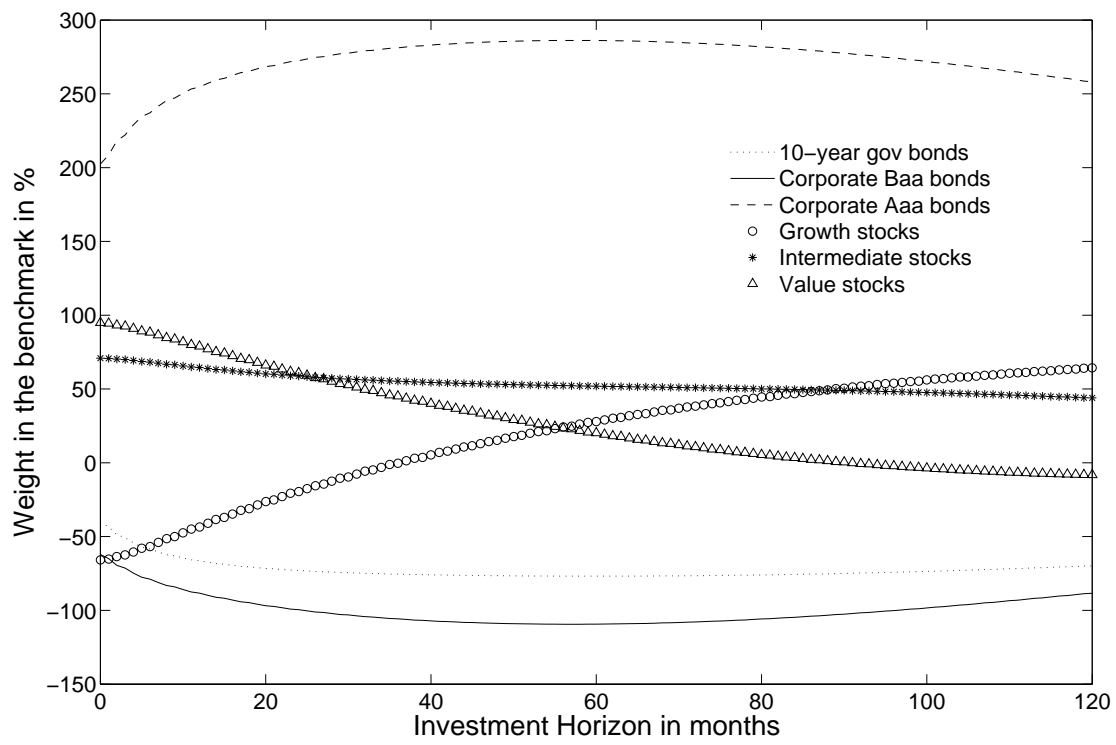

Panel B: Optimal strategic asset allocation of the CIO

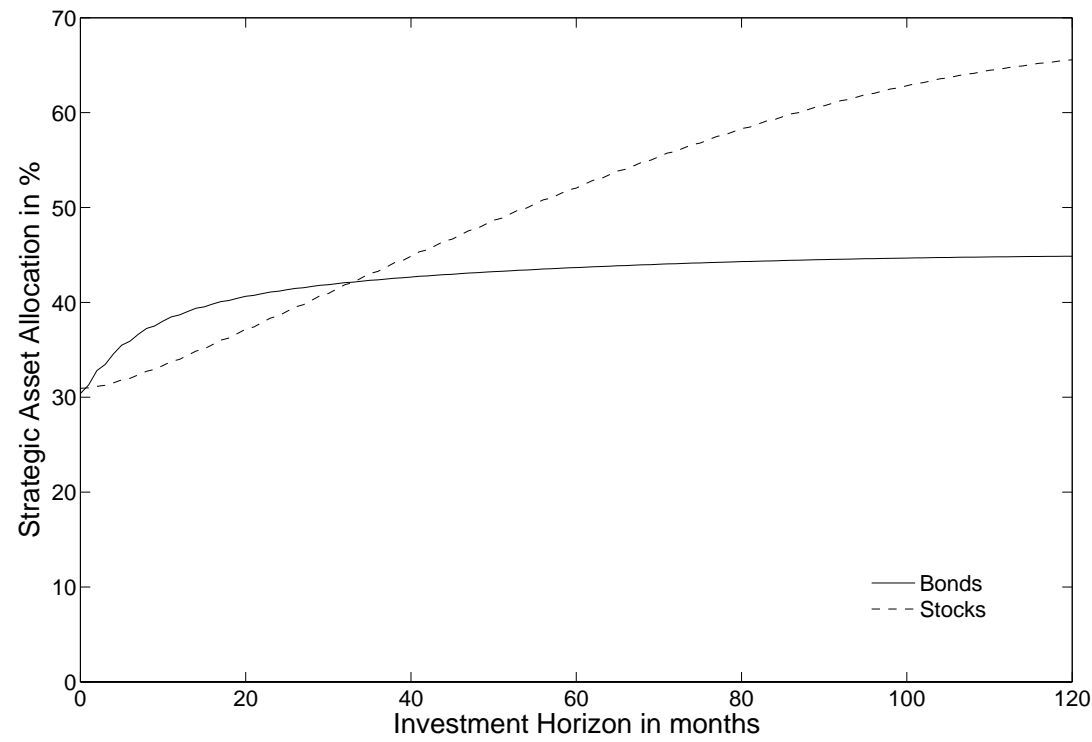

Figure 10: Optimal performance benchmarks and strategic allocation. Panel A portrays the composition of the optimal performance benchmarks for different investment horizons of the CIO. Panel B presents the corresponding optimal strategic asset allocation to the asset classes. We plot the benchmark for the stock and bond manager in the same graph, but there is still no cross-benchmarking. I.e., the benchmark weights in both asset classes each sum up to 100 percent. The horizontal axis depicts the investment horizon of the CIO in months. The preference parameters have been set to $\gamma_{C}=10$ and $\gamma_{i}=5$, with $i=1,2$. 
Panel A: The investment horizon of the CIO equals $T=1$

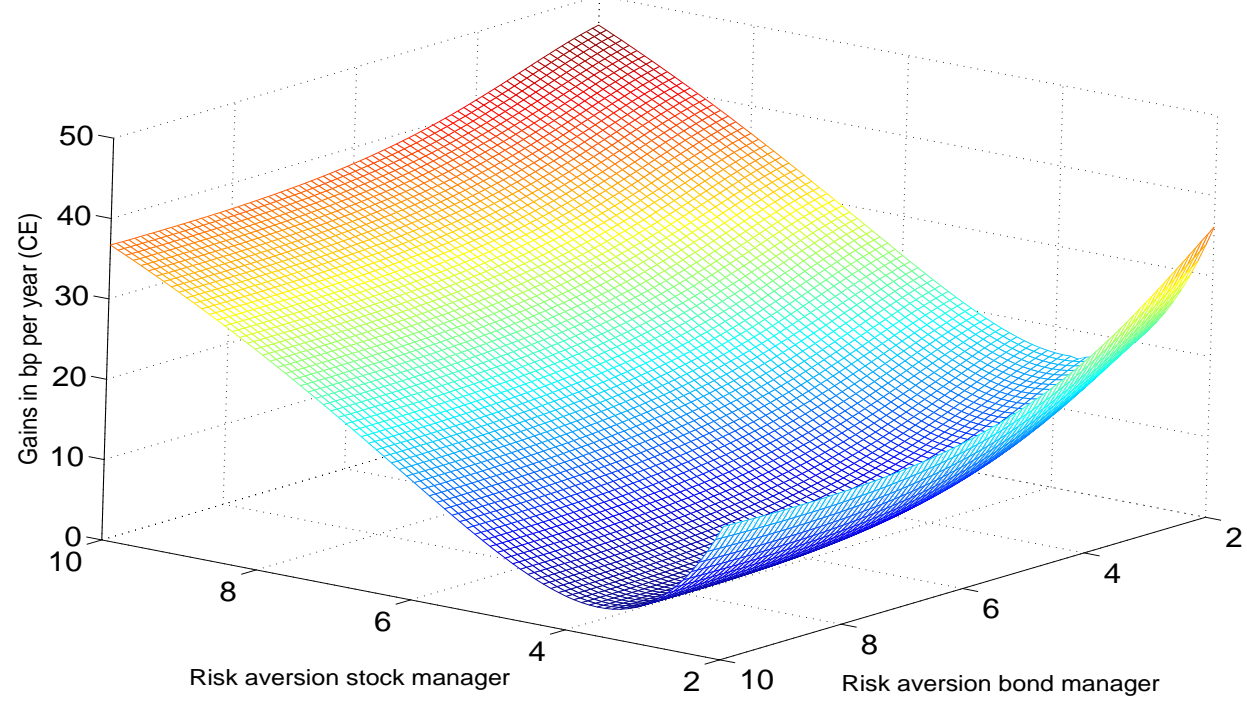

Panel B: The investment horizon of the CIO equals $T=10$

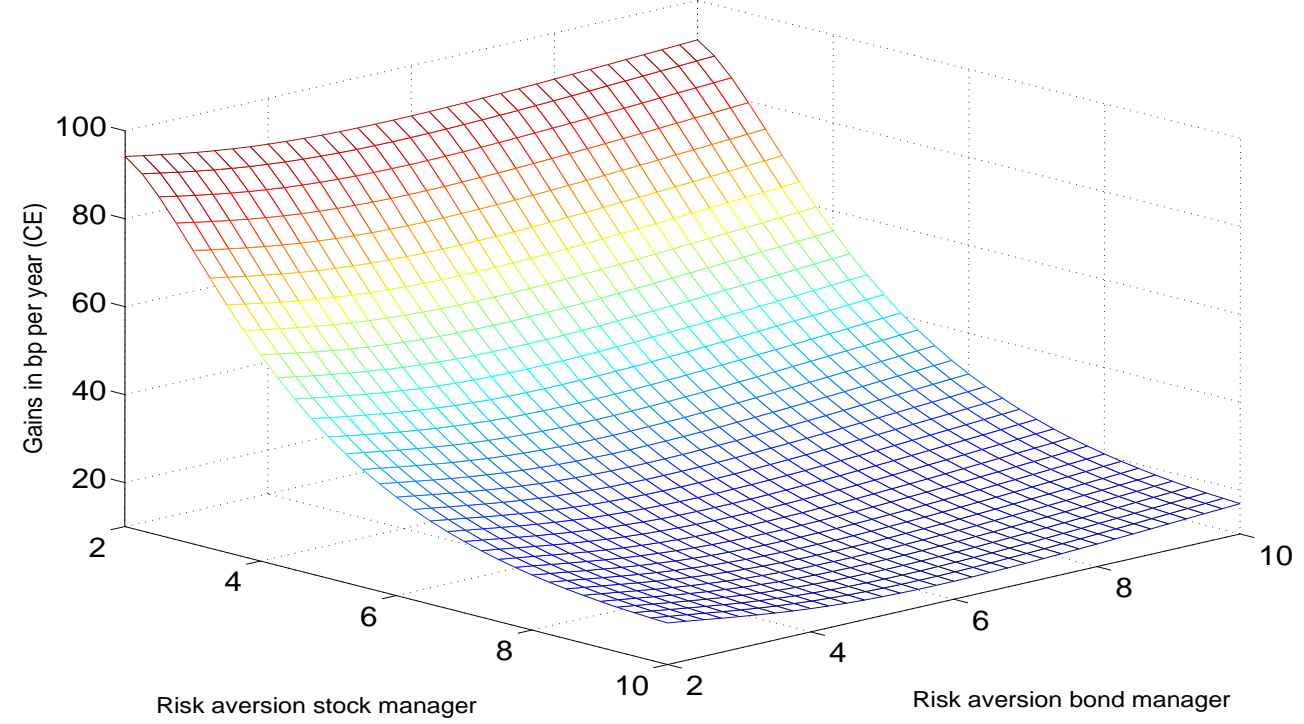

Figure 11: Value generated by an optimally chosen benchmark. Comparison of certainty equivalents following from the decentralized with and without an optimally chosen benchmark. We present the annualized gains in basis points from using the benchmark optimally. The investment horizon of the CIO equals one year in Panel A and 10 years in Panel B. The $\mathrm{x}$ - and $\mathrm{y}$-axis depict different risk attitudes of the asset managers. The coefficient of relative risk aversion of the CIO equals 10. 
Panel A: The investment horizon of the CIO equals $T=1$

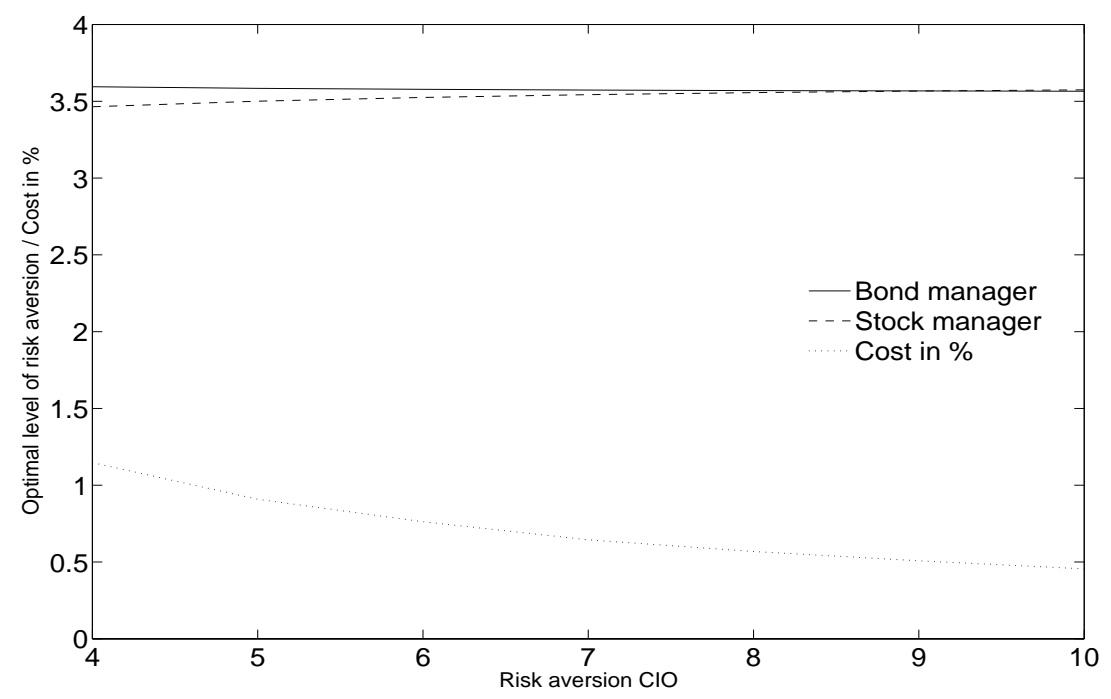

Panel B: The investment horizon of the CIO equals $T=10$

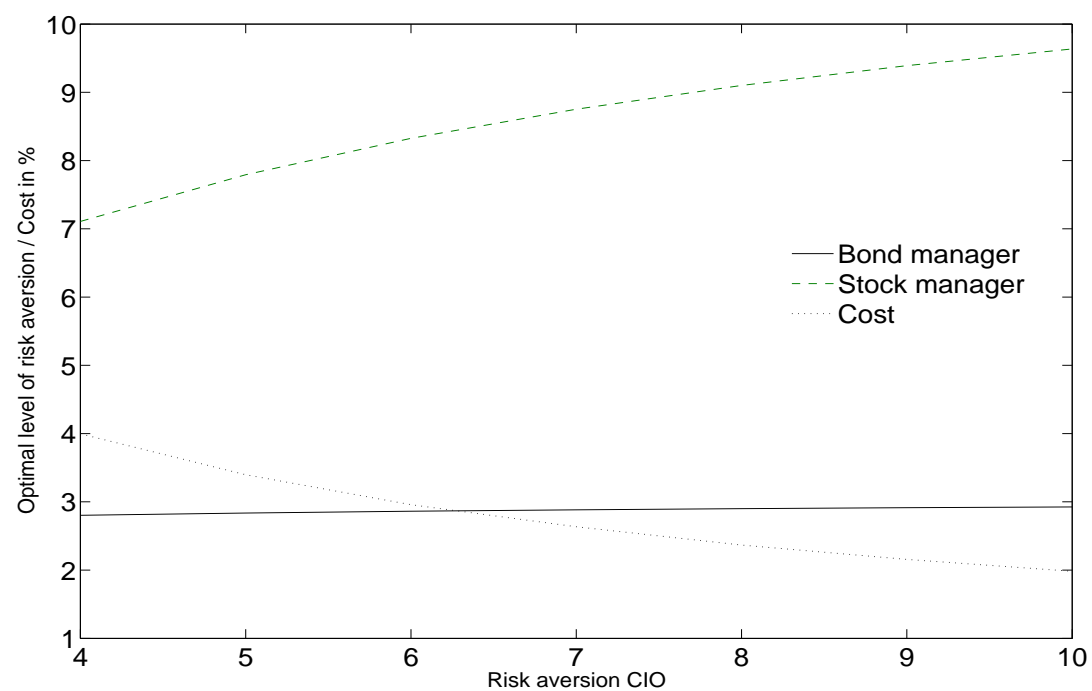

Figure 12: Optimal selection of investment managers. Optimal risk aversion level (from the perspective of the CIO) of the bond and stock manager for different risk attitudes of the CIO. The investment horizon $T$ equals one year in Panel A and 10 years in Panel B. The horizontal axis depicts the coefficient of relative risk aversion of the CIO. The cost is computed by taking the ratio of the certainty equivalents achieved under centralized and decentralized investment management after which we subtract one and multiply by -100 to express the cost in percent per year. 

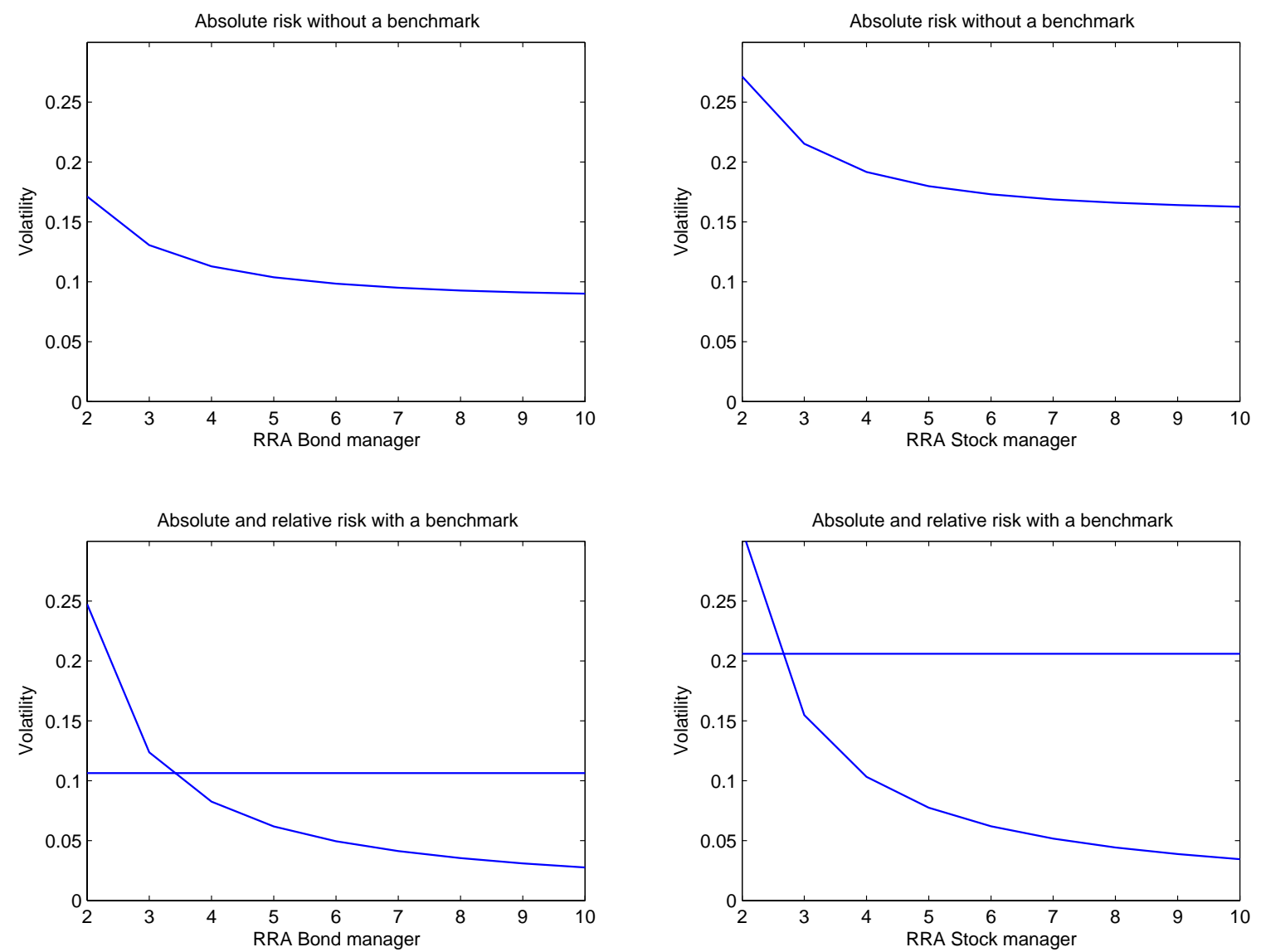

Figure 13: Implied absolute and relative risk levels. The volatility of the portfolio return (absolute risk) and the volatility of the portfolio return in excess of the optimally designed benchmark return (relative risk) are provided. The CIO has a risk aversion of $\gamma_{C}=10$, while the risk attitudes of the managers are given by $\gamma_{1}=\gamma_{2}=5$. The two top graphs provide the absolute risks of the portfolios in absence of a performance benchmark. The two bottom graphs portray the relative risks (downward sloping) and absolute risks (constant) when the managers operate in the presence of a benchmark. The left graphs correspond to the bond manager. The right graphs correspond to the stock manager. 
Panel A: The coefficient of relative risk aversion of the CIO equals $\gamma_{C}=5$

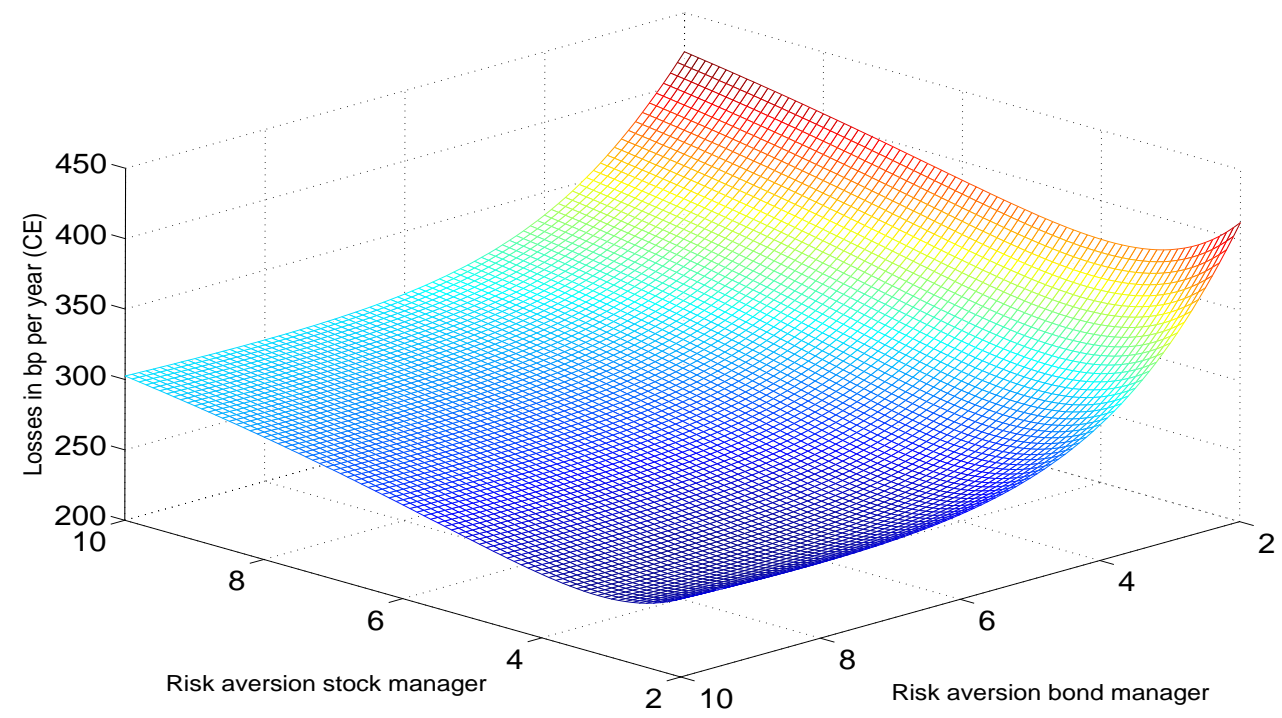

Panel B: The coefficient of relative risk aversion of the CIO equals $\gamma_{C}=10$

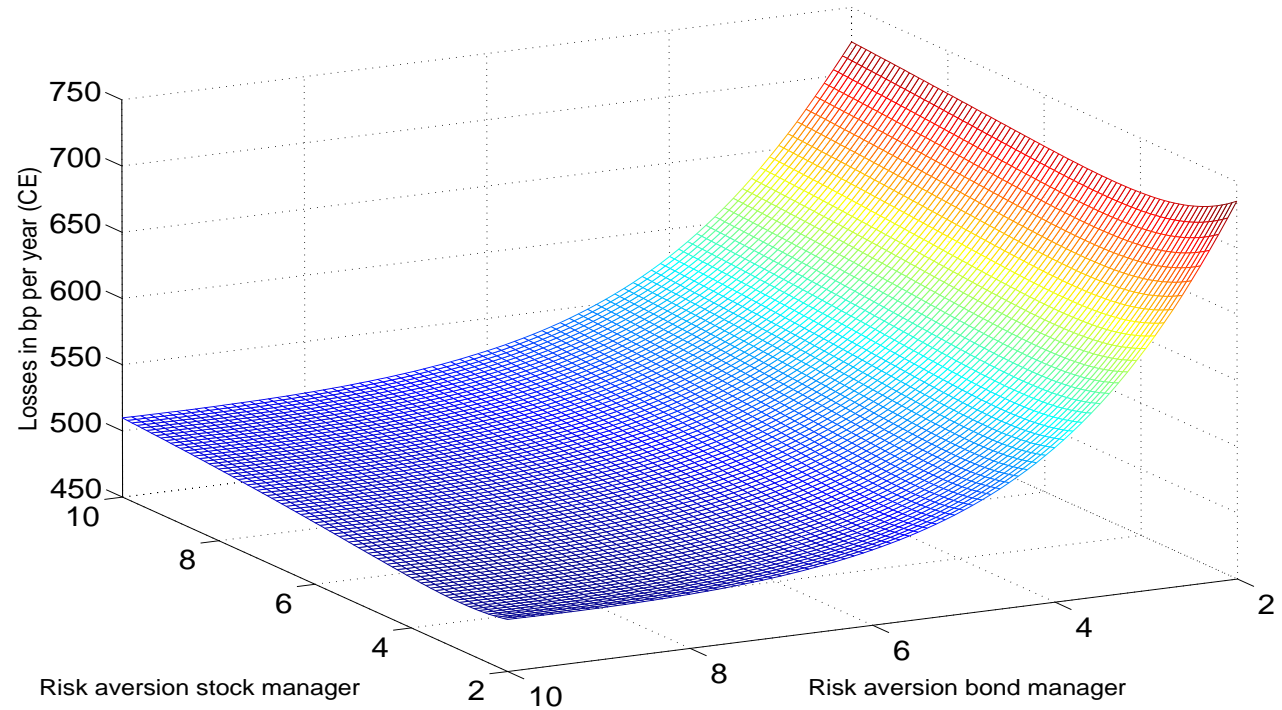

Figure 14: Utility costs of decentralized investment management in the presence of liabilities. Utility costs in terms of certainty equivalents of decentralized investment management in absence of a performance benchmark but in the presence of liabilities. The coefficient of relative risk aversion of the CIO is given by $\gamma_{C}=5$ in Panel A and $\gamma_{C}=10$ in Panel B. The losses are computed by taking the ratio of the certainty equivalents achieved under centralized and decentralized investment management after which we subtract one and multiply by $-10,000$ to express the losses in basis points per year. 
Panel A: Model parameters

\begin{tabular}{|c|c|c|c|c|c|c|c|c|}
\hline Source of risk & $Z_{1}$ & $Z_{2}$ & $Z_{3}$ & $Z_{4}$ & $Z_{5}$ & $Z_{6}$ & & \\
\hline$\Lambda$ & 0.331 & 0.419 & -0.0291 & 0.126 & 0.477 & 0.305 & & \\
\hline$\Sigma$ & & & & & & & & \\
\hline Gov. bonds & $13.5 \%$ & 0 & 0 & 0 & 0 & 0 & & \\
\hline Corp. bonds, Baa & $8.2 \%$ & $5.6 \%$ & 0 & 0 & 0 & 0 & & \\
\hline Corp. bonds, Aaa & $9.1 \%$ & $2.7 \%$ & $2.4 \%$ & 0 & 0 & 0 & & \\
\hline Growth stocks & $3.7 \%$ & $6.3 \%$ & $0.3 \%$ & $16.5 \%$ & 0 & 0 & & \\
\hline Int. stocks & $3.6 \%$ & $6.8 \%$ & $0.3 \%$ & $11.7 \%$ & $7.3 \%$ & 0 & & \\
\hline Value stocks & $3.6 \%$ & $7.7 \%$ & $0.1 \%$ & $10.4 \%$ & $6.8 \%$ & $5.9 \%$ & & \\
\hline \multicolumn{9}{|c|}{ Panel B: Implied parameters } \\
\hline & \multicolumn{4}{|c|}{ Expected return } & \multicolumn{3}{|c|}{ Correlation } & \\
\hline Gov. bonds & \multicolumn{2}{|c|}{$9.5 \%$} & $100 \%$ & $82 \%$ & $93 \%$ & $20 \%$ & $23 \%$ & $22 \%$ \\
\hline Corp. bonds, Baa & \multicolumn{2}{|c|}{$10.1 \%$} & $82 \%$ & $100 \%$ & $92 \%$ & $37 \%$ & $43 \%$ & $45 \%$ \\
\hline Corp. bonds, Aaa & \multicolumn{2}{|c|}{$9.1 \%$} & $93 \%$ & $92 \%$ & $100 \%$ & $29 \%$ & $34 \%$ & $34 \%$ \\
\hline Growth stocks & \multicolumn{2}{|c|}{$10.9 \%$} & $20 \%$ & $37 \%$ & $29 \%$ & $100 \%$ & $88 \%$ & $80 \%$ \\
\hline Int. stocks & \multicolumn{2}{|c|}{$14.0 \%$} & $23 \%$ & $43 \%$ & $34 \%$ & $88 \%$ & $100 \%$ & $93 \%$ \\
\hline Value stocks & \multicolumn{2}{|c|}{$15.7 \%$} & $22 \%$ & $45 \%$ & $34 \%$ & $80 \%$ & $93 \%$ & $100 \%$ \\
\hline
\end{tabular}

Table 1: Estimation results for the financial market in Section 2. Estimation results of the financial market in Section 2 over the period January 1973 through November 2004 using monthly data. The model is estimated by maximum likelihood. The asset set contains government bonds ('Gov. bonds'), corporate bonds with credit ratings Baa ('Corp. bonds, Baa') and Aaa ('Corp. bonds, Aaa'), and three equity portfolio ranked on their book-to-market ratio (growth/intermediate ('Int. ')/value). Panel A provides the model parameters and Panel B portrays the implied instantaneous expected returns $(r+\Sigma \Lambda)$ and correlations. In determining $\Lambda$, we assume that the instantaneous nominal short rate equals $r=5 \%$. 


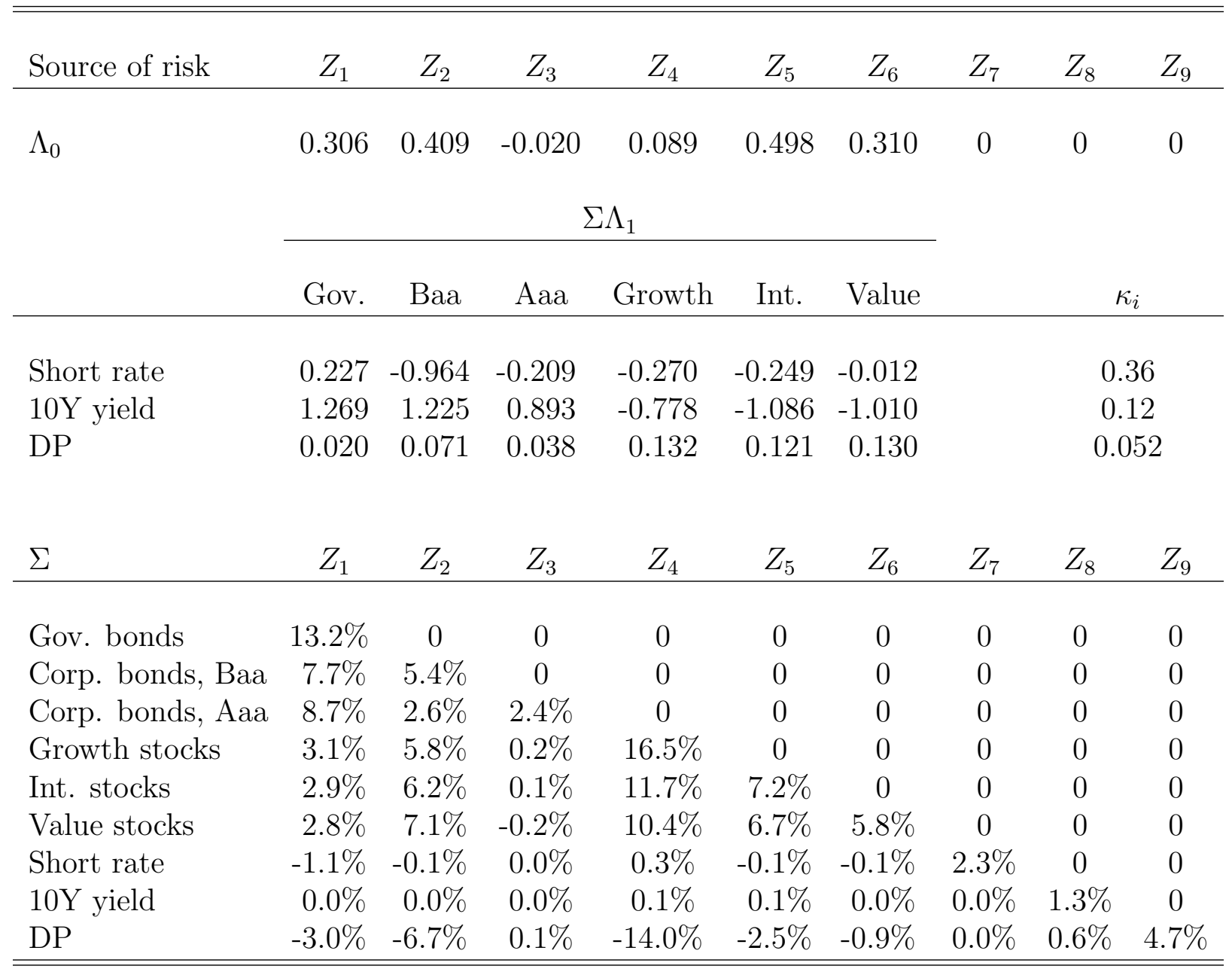

Table 2: Estimation results for the financial market in Section 3. Estimation results of the financial market in Section 3 over the period January 1973 through November 2004 using monthly data. The model is estimated by maximum likelihood. The asset set contains government bonds ('Gov. bonds'), corporate bonds with credit ratings Baa ('Corp. bonds, Baa') and Aaa ('Corp. bonds, Aaa'), and three equity portfolio ranked on their book-to-market ratio (growth/intermediate ('Int. ')/value). In determining $\Lambda_{0}$, we assume that the instantaneous nominal short rate equals $r=5 \%$. We report $\Sigma \Lambda_{1}$ rather than $\Lambda_{1}$ as the former expression is easier to interpret. The short rate, the yield on a $10 \mathrm{Y}$ nominal government bond, and the dividend yield are used to predict returns. 


\begin{tabular}{|c|c|c|c|c|c|c|}
\hline \multirow[b]{2}{*}{$\gamma_{C}$} & \multicolumn{2}{|c|}{ Managers only } & \multicolumn{2}{|c|}{ Managers/benchmark } & \multicolumn{2}{|c|}{ Value benchmark } \\
\hline & $T_{C}=1$ & $T_{C}=10$ & $T_{C}=1$ & $T_{C}=10$ & $T_{C}=1$ & $T_{C}=10$ \\
\hline 4 & 114.7 & 399.6 & 80.5 & 354.6 & $34.6(30 \%)$ & $46.9(12 \%)$ \\
\hline 5 & 90.9 & 339.7 & 63.8 & 301.3 & $27.3(30 \%)$ & $39.7(12 \%)$ \\
\hline 6 & 76.2 & 295.8 & 53.2 & 263.2 & $23.2(30 \%)$ & $33.6(11 \%)$ \\
\hline 7 & 64.5 & 263.4 & 44.8 & 234.6 & $19.8(31 \%)$ & $29.7(11 \%)$ \\
\hline 8 & 56.8 & 236.7 & 39.7 & 211.8 & $17.2(30 \%)$ & $25.6(11 \%)$ \\
\hline 9 & 50.8 & 215.7 & 35.4 & 193.1 & $15.5(31 \%)$ & $23.1(11 \%)$ \\
\hline 10 & 45.6 & 198.2 & 31.9 & 177.1 & $13.7(30 \%)$ & $21.5(11 \%)$ \\
\hline
\end{tabular}

Table 3: Costs of decentralization with optimally selected managers and benchmarks. Costs of decentralized investment management for the financial market in Section 3. The first two columns provide the costs (in basis points) when the CIO only optimizes over the risk attitudes of the investment managers. Column 3 and 4 portray the costs of decentralized investment management when the CIO optimizes over the risk attitudes of investment managers and their respective benchmarks. The last two columns present the value (in basis points and as a fraction of total costs in parentheses) of introducing a performance benchmark. All results are determined for the CIO's investment horizon equal to $T_{C}=1$ and $T_{C}=10$ and coefficients of risk aversion ranging from $\gamma_{C}=4$ up to $\gamma_{C}=10$. 